\title{
Use of Oral Contraceptives as a Potential Risk Factor for Breast Cancer: A Systematic Review and Meta-Analysis of Case-Control Studies Up to 2010
}

\author{
Wiesław Kanadys ${ }^{1}$, Agnieszka Barańska ${ }^{2}$, Maria Malm ${ }^{2, *}$,, Agata Błaszczuk $^{3}$, Małgorzata Polz-Dacewicz ${ }^{3}$, \\ Mariola Janiszewska ${ }^{2}$ (I) and Marian Jędrych ${ }^{2}$ (I) \\ 1 Specialistic Medical Center "Czechów” in Lublin, 20-848 Lublin, Poland; wieslaw.kanadys@wp.pl \\ 2 Department of Medical Informatics and Statistics with E-learning Lab, Medical University, \\ 20-090 Lublin, Poland; agnieszkabaranska@umlub.pl (A.B.); mariola.janiszewska@umlub.pl (M.J.); \\ marian.jedrych@umlub.pl (M.J.) \\ 3 Department of Virology with SARS Laboratory, Medical University, 20-093 Lublin, Poland; \\ agata.blaszczuk@umlub.pl (A.B.); malgorzata.polz.dacewicz@umlub.pl (M.P.-D.) \\ * Correspondence: maria.malm@umlub.pl
}

Citation: Kanadys, W.; Barańska, A.; Malm, M.; Błaszczuk, A.; Polz-Dacewicz, M.; Janiszewska, M.; Jęrych, M. Use of Oral Contraceptives as a Potential Risk Factor for Breast Cancer: A Systematic Review and Meta-Analysis of Case-Control Studies Up to 2010. Int. J. Environ. Res. Public Health 2021, 18, 4638. https://doi.org/10.3390/ijerph18094638

Academic Editor: Claudio Costantino

Received: 12 March 2021

Accepted: 21 April 2021

Published: 27 April 2021

Publisher's Note: MDPI stays neutra with regard to jurisdictional claims in published maps and institutional affiliations.

Copyright: (c) 2021 by the authors. Licensee MDPI, Basel, Switzerland. This article is an open access article distributed under the terms and conditions of the Creative Commons Attribution (CC BY) license (https:/ / creativecommons.org/licenses/by/ $4.0 /)$.

\begin{abstract}
Despite numerous studies evaluating the risk of breast cancer among oral contraception users, the effect of oral contraceptive on developing breast cancer remains inconclusive. Therefore, we conducted a systematic review of literature with meta-analysis in order to quantitative estimate this association. The bibliographic database MEDLINE and EMBASE, and reference lists of identified articles were searched, with no language restrictions, from the start of publication to August 2010. We performed a reanalysis and overall estimate of 79 case-control studies conducted between 1960-2010, including a total of 72,030 incidents, histologically confirmed cases of breast cancer and 123,650 population/hospital controls. A decrease was observed in cancer risk in OC users before age 25 years $(0.91,0.83-1.00)$. However, the use of OCs before the first full-term pregnancy had a significant increased risk of breast cancer (OR, 1.14, 1.01-1.28, $p=0.04)$, as did OC use longer than 5 years $(1.09,1.01-1.18, p=0.02)$. Pooled crude odds ratios of breast cancer in ever-users of oral contraceptives was 1.01 [95\% confidence interval (CI), 0.95-1.07], compared with never-users. There was no significant increase in risk among premenopausal women $(1.06,0.92-1.22)$, postmenopausal women $(0.99,0.89-1.10)$, or nulliparous women $(1.02,0.82-1.26)$. Oral contraceptives do not appear to increase the risk of breast cancer among users. However, OC use before a first full-term pregnancy or using them longer than 5 years can modify the development of the breast cancer.
\end{abstract}

Keywords: breast cancer; cancer risk; prevention; oral contraceptives; meta-analysis

\section{Introduction}

Breast cancer $(\mathrm{BrCa})$ is the most frequently diagnosed cancer and the leading cause of cancer death in women worldwide. It accounts for $23 \%$ (1.38 million) of the total new cancer cases and $14 \%(458,400)$ of the total deaths in 2008. About half the BrCa cases and $60 \%$ of the deaths occur in economically developing countries [1]. Of every thousand women aged 50, two will recently have given a diagnosis of $\mathrm{BrCa}$, and about 15 will have had a diagnosis made before age 50, giving a prevalence of $\mathrm{BrCa}$ of nearly $2 \%$ [2].

In Western countries, the incidence has increased BrCa over the last 50 years, and these increases were dramatic in both the 1980s and the 1990s [3,4]. The sharp incidence increases observed after 1985 in the United States and most European countries were mainly due to the combined effects of changes in reproductive factors, prevalence of overweight and obesity, improvements screening mammography, and use of hormone replacement therapy (HRT) [5]. After peaking in 2000, the incidence of invasive BrCa steadily decreased, as did the mortality rate [6,7]. The potential explanation for the observed decrease in incidence 
may be the decline in the use of HRT after the publication of the results of the Women's Health Initiative study in 2002 [7,8]; the early detection and management of invasive and precancerous breast lesions and the subsequent increase in management of these lesions with selective estrogen receptor modulators; and observed changes in lifestyle, such as increased exercise, decreased obesity, and dietary habits [9]. It may also be the result of the recently observed reduction in mammographic screening, which may lead to a decrease in $\mathrm{BrCa}$ detection among unscreened women [10].

The etiology BrCa is complex. Susceptibility is influenced by both environmental and genetic factors. In $5-10 \%$ of the cases, BrCa has a hereditary basis. BrCa susceptibility is generally inherited as an autosomal dominant with limited penetrance. In $15-30 \%$ of patients from high-risk families, $\mathrm{BrCa}$ is caused by a germline mutation in the BRCA1 or BRCA2 gene [11]. Many risk factors are associated with the development of $\mathrm{BrCa}$, but the causal mechanism cannot be directly linked to a single one of them which include: age, familial history of $\mathrm{BrCa}$, personal history of benign breast biopsy (atypical ductal and lobular hyperplasia- $\mathrm{ADH}$ and $\mathrm{ALH}$ ), breast density, factors associated with lifestyle (cigarette smoking, alcohol use, dietary fat intake, and postmenopausal obesity), and reproductive factors (nulliparity, late age of first live birth) [2]. Experimental and epidemiological evidence suggests that exposure to endogenous hormones, notably estrogens and androgens, promotes breast carcinogenesis. Factors related to increased estrogen exposure throughout a woman's lifetime, such as early menarche, late menopause, use of oral contraceptives, and hormone replacement therapy, have been associated with a $\sim 2$-fold increase in $\mathrm{BrCa}$ risk among women $[12,13]$.

Oral contraceptives (OCs) among the most widely used methods are of effective and reversible family planning [14]. Many questions exist concerning a possible association between the use of OCs and the development of cancer. Benefits and risks of OCs use on cancer were reviewed by Working Groups of the International Agency for Research on Cancer (IARC/WHO) in 2007, which concluded that OC are carcinogenic to humans [15]. OCs use has been associated with an excess risk of benign liver tumors and modest increased risk of liver cancer and cervical cancer. Simultaneously OC protect again the risk of ovarian and endometrial cancer. No association is present between OC use and lung, other digestive tract neoplasms, cutaneous malignant melanoma, thyroid cancer, and any of the other neoplasms investigated [16].

Despite the large number of studies in this area, no consensus is present regarding the exact effect of OCs on the risk of $\mathrm{BrCa}[9,17]$. In this context, we sought to evaluate whether women using oral contraceptives exhibit an increased risk of BrCa by performing a systematic review and meta-analysis of the relevant data from available case-control studies.

\section{Materials and Methods}

\subsection{Search Strategy}

We systematically searched the PubMed (Medline) and EMBASE bibliographic databases (from their commencements to August 2010), with no language restriction, to identify studies investigating the relation between $\mathrm{OC}$ uses and the risk of BrCa developing. For computer searches, we used the following MeSH terms or keywords: 'breast cancer', 'breast malignancy' or 'breast neoplasm', combined with 'oral contraceptives', 'contraceptive agents' or 'birth control pill'. In addition, we examined the references of identified articles, previous review articles and meta-analysis, and other relevant publications to identify further pertinent studies [18-22].

\subsection{Study Selection}

We selected studies in a two-stage process. Initially, a preliminary selection of articles was made after the electronic searching of titles. When the citation was relevant or when title/abstract was not sufficient for inclusion/exclusion, full-texts were retrieved and evaluated. Secondly, full manuscripts of selected citation and the additional items derived from a review of literature of selected papers and review articles were obtained. Final 
inclusion/exclusion decisions were made after independent and duplicate examination of the full manuscripts.

Studies were included in the systematic review if they were a case-control design (population- and hospital-based) and published as original articles that permitted assessment of an association between the use of OCs and risk of BrCa. Studies eligible for meta-analysis fulfilled the following criteria: including at least 100 women with incident invasive $\mathrm{BrCa}$, and presenting sufficient detailed results to calculate the odds ratio (OR) and $95 \%$ confidence interval (CI). In the case of multiple published studies based on the same population or subpopulation, we took into account only the most recent published report from the study with the longest period of recruitment, unless the earlier reports contained information not available in subsequent reports or represented a comprehensive form of this study. The selection procedure for studies included in meta-analysis is presented in Figure 1.
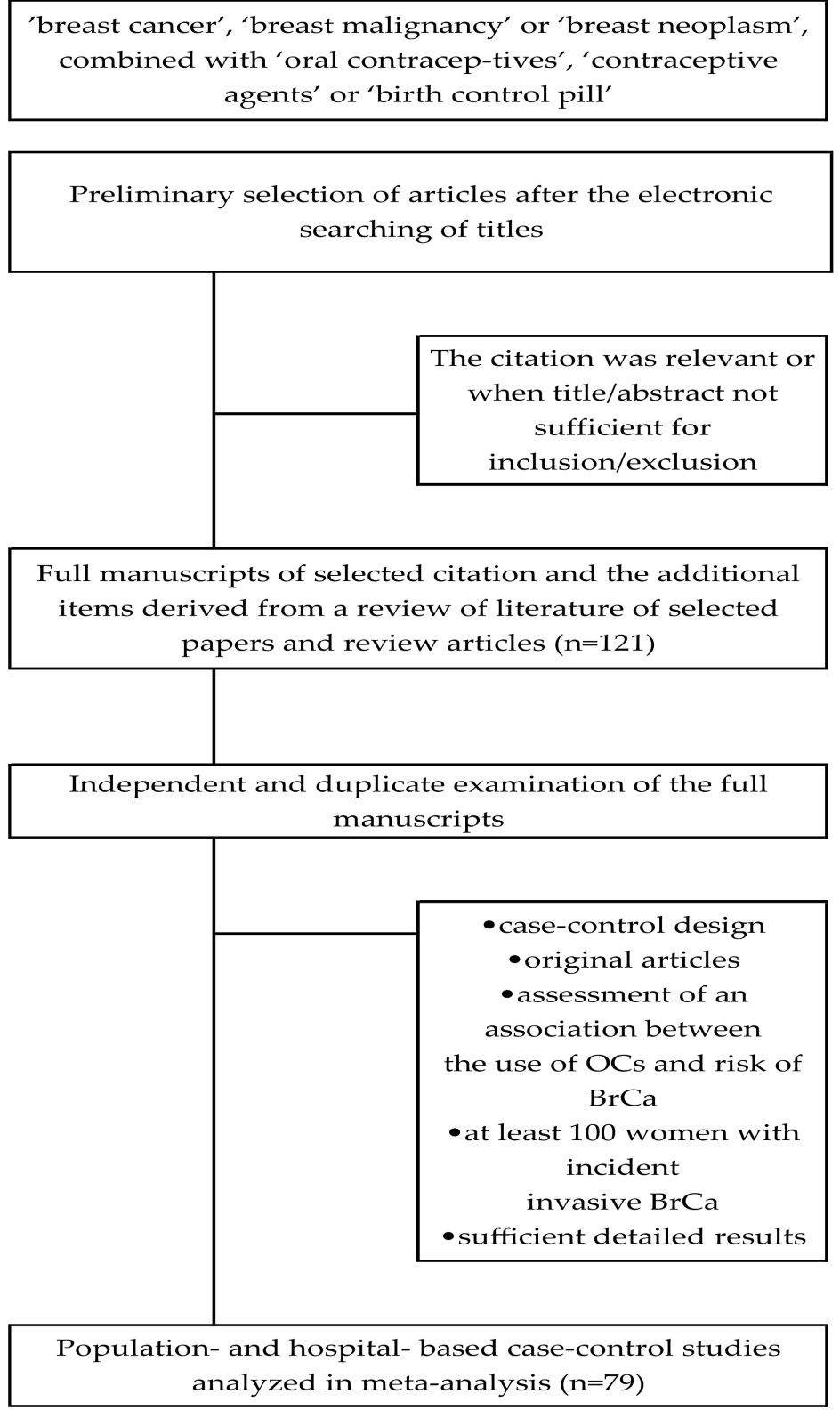

Figure 1. Flowchart of the selection procedure for studies included in meta-analysis regarding use of oral contraceptives as a potential risk factor for breast cancer. 


\subsection{Data Extraction}

The following data were extracted for each study: (a) clinical and methodological study characteristics such as last name of first author, publication year, country in which the study was performed, name of the study, years of data collection, number of cases and controls subjects, and source of cases; (b) information on use of OC (exposure)-ever/never use, duration of use at least above 5 years, use before first full-term pregnancy, and use in different age groups ( $<25$ years, premenopausal, and postmenopausal women). To summarize the results associated with menopausal status, we adopted the following definitions: (a) premenopausal women or women younger than 50 years and (b) postmenopausal women or women older than 50 years. To assess the risk of developing breast cancer associated with the use of oral contraceptives with different composition (high- versus low formulation), we classified the available studies (based on the period of recruitment) to the following time ranges: 1966-1985 and 1986-2010 [23-26]. We additionally conducted a separate meta-analysis to determine whether the size of the risk of breast cancer is affected by the type of control group, based on the neighborhood/general population or on hospital.

If the study presented data on breast cancer only in subpopulations according to different ethnicity, age group, parity, etc., we conducted a cumulative estimate of these data for the entire study population. When the breast cancer event rate, OC use rate or control recruitment rate was expressed as a percentage, the number of patients was calculated by multiplying the rate by the total number of women in the relevant group. When data were expressed in a way that did not allow exact figures to be obtained, these data were not included in the meta-analysis.

\subsection{Assessment of Study Quality}

The Newcastle-Ottawa Scale (NOS) score was employed to evaluate the study quality of observational studies, with a maximum score of 9, of which 0-3, 4-6, and 7-9 scores were considered as low, fair, and high quality, respectively [27]. With this tool, each study in the meta-analysis was assessed in three separate categories: selection of cases and controls, comparability of cases and controls on the basis of the design or analysis, and ascertainment of exposure. The NOS quality stars ranged between $5-7$, and the average score was 5.5 for case-control studies (Supplementary Materials File S1). Seven (7.86\%) studies were regarded as high quality (NOS $\geq 7$ points).

\subsection{Statistical Methods}

Meta-analysis of summary statistics from individual studies was performed utilizing the Medical Package program of STATISTICA 11.0 software (StatSoft Poland, Krakow, Poland). For each study, we constructed separate two-by-two $(2 \times 2)$ contingency tables to calculate the odds ratios (OR) and 95\% confidence intervals (CIs), cross-classifying OC users and occurrence of breast cancer. The Mantel-Haenszel test was calculated to assess the association between OC use and cancer. An OR of one indicates that the odds of having breast cancer are the same in the case group and the control group; an OR greater than one indicates that the odds of having breast cancer in the case group are greater than in the control group; an OR of less than one indicates that the odds of having breast cancer in the control group are greater than in the case group. Because about one-third of the studies did not present adjusted ORs, only crude ORs were used in the primary meta-analysis. Meta-analyses combining the ORs across studies were conducted using the DerSimonianLaird random effects model [28]. The random effects meta-analysis model was applied due to the diversity of research in terms of, for example, design and population. In the random effects model, it is assumed that there is no common effect size for independent studies. Instead, each study is assumed to have a different population effect size, which is a random variable and has a normal distribution. Therefore, there is a difference between the size of the effects of individual studies. Thus, the variance of the effect size in the random effects model is the sum of the variance within and between studies. As suggested by DerSimonian and Laird [28], the variance 'between studies' is estimated using the moments 
method. Weighting of the studies in the meta-analysis was calculated on the basis of the inverse of the sum of 'within study' and between studies variances. Heterogeneity was assessed graphically using forest plots and statistically using the $\mathrm{Q}$ test and I2 index. I2 values of $25 \%, 50 \%$, and $75 \%$ were regarded as respectively representing low, moderate, and high heterogeneity between studies $[29,30]$.

Due to the high heterogeneity of the studies, the following subgroups were also analyzed: case-control studies of the period of recruitment into the study before 1986/casecontrol studies of the period of recruitment into the study after 1986; premenopausal women or women younger than 50 years/postmenopausal women or women older than 50 years/women under 25 years old; nulliparous women; ever use of oral contraceptives before first pregnancy; and ever use of oral contraceptives for longer than 5 years.

For all the analyses, forest plots were generated to display results, whereby diamonds represent study-specific odds ratios and $95 \%$ CIs for individual studies are represented by horizontal lines.

To analyze the publication bias, the Begg and Mazumdar and Egger tests were performed. The meta-analysis methodology was based on the guidelines of DerSimonian and Laird [28] and Higgins et al. [29,30]. The method used is globally recognized as the primary method for the evaluation of case-control studies.

\section{Results}

Our systematic review included 121 population- and hospital-based case-control studies that analyzed the relationship between use of oral contraceptives and histologically confirmed breast cancer. Of these, 79 that met inclusion criteria qualified for the meta-analysis [31-109].

\subsection{Description of the Studies}

Table 1 shows papers in chronological order of recruitment period. Study periods included years between 1960-2010. All papers were published in English, except for one in Portuguese [95]. The total sample included 72,030 women with breast cancer and 123,650 women without breast cancer; respectively, of this number, 32,326 women (44.8\%) and 53,365 women (43.2\%) ever using combined oral contraceptives. North American studies were most common $(n=30)$, followed by 20 from Europe, 9 from Asia-Pacific, 7 from West Asia, 5 from Australia/New Zealand, 3 from Africa, 2 from South America, and 1 from Central America. One study was conducted in Sweden and Norway [68], and the WHO Collaborative Study of Neoplasia and Steroid Contraceptives [58] analyzed multinational data (participating countries: Australia, Chile, Republic of China, Colombia, the German Democratic Republic, Israel, Kenya, Mexico, Philippines and Thailand). In 39 studies, cases sources were regional cancer registries, while in the remaining 40 studies, hospital data was used as the case source. Forty studies were based on neighborhood/general population control group, 32 studies based on hospital control groups, and 6 studies were based on clinic control groups. One study used a combination of hospital and population controls [95]. 
Table 1. Characteristics of the case-control studies included in the meta-analysis on the association between oral contraceptive use and breast cancer risk

\begin{tabular}{|c|c|c|c|c|c|c|c|c|c|}
\hline $\begin{array}{c}\text { Study Name } \\
\text { Setting [Reference] }\end{array}$ & Recruitment Period & $\begin{array}{l}\text { Number of } \\
\text { Case Subjects }\end{array}$ & $\begin{array}{l}\text { Percent of } \\
\text { OCs Users }\end{array}$ & $\begin{array}{c}\text { Age Range } \\
\text { (Years) }\end{array}$ & $\begin{array}{l}\text { Source of } \\
\text { Cases }\end{array}$ & $\begin{array}{l}\text { Number of } \\
\text { Controls Subjects }\end{array}$ & $\begin{array}{l}\text { Percent of } \\
\text { OCs Users }\end{array}$ & $\begin{array}{l}\text { Source of } \\
\text { Controls }\end{array}$ & NOS Sc. \\
\hline USA-Nationwide [31] & 1960-1976 & 989 & 27.6 & $30-55$ & Population & 9901 & 26.7 & Population & 5 \\
\hline UK-London, Oxford [32] & December 1968-September 1980 & 1176 & 45.7 & $16-50$ & Hospital & 1176 & 47.1 & Hospital & 5 \\
\hline USA-New York City [33] & January 1969-December 1975 & 518 & 13.9 & $30<$ & Hospital & 1601 & 18.0 & Hospital & 5 \\
\hline USA-Baltimore [34] & 1969-1972 & 284 & 7.7 & $20-74$ & Hospital & 367 & 9.3 & Hospital & 5 \\
\hline USA-San Francisco Bay Area [35] & January 1970-December 1972 & 1868 & 28.7 & $15-49+$ & Hospital & 3391 & 29.6 & Hospital & 5 \\
\hline Canada_northern Alberta [36] & January 1971-December 1974 & 295 & 35.6 & $30-49$ & Hospital & 507 & 47.1 & Clinic & 5 \\
\hline former Yugoslavia_Slovenia [37] & May 1972-November 1974 & 190 & 15.8 & $20-49$ & Hospital & 380 & 17.1 & Clinic & 5 \\
\hline USA-Los Angeles County [38] & July 1972-December 1978 & 163 & 82.8 & $<33$ & Population & 270 & 80.7 & Population & 5 \\
\hline $\begin{array}{l}\text { Breast Cancer Detection Demonstration } \\
\text { Project USA-Nationwide [39] }\end{array}$ & July 1973-November 1980 & 2022 & 23.8 & $<40-60+$ & Population & 2183 & 24.5 & Population & 5 \\
\hline USA—New York State [40] & January 1974-August 1976 & 253 & 52.6 & $\leq 45$ & Population & 497 & 46.9 & Population & 5 \\
\hline Canada_northern Alberta [41] & 1976-1977 & 577 & 26.2 & $30-80$ & Population & 826 & 42.3 & Population & 5 \\
\hline USA-North Carolina [42] & April 1977-December 1978 & 158 & 41.8 & $25-59$ & Hospital & 1140 & 48.4 & Population & 5 \\
\hline \multirow{2}{*}{$\begin{array}{c}\text { Case-Control Surveillance Study } \\
\text { USA-Philadelphia. Baltimore, New } \\
\text { York City [44,45] }\end{array}$} & \multirow{2}{*}{ 1977-1992 } & 3540 & 31.1 & $25-59$ & Hospital & 4488 & 31.6 & Hospital & 7 \\
\hline & & 519 & 31.0 & $25-59$ & Hospital & 1008 & 27.3 & Hospital & 5 \\
\hline Brazil—Belo Horizonte [46] & January 1978-December 1987 & 293 & 20.5 & $25-75$ & Hospital & 566 & 14.5 & Hospital & 5 \\
\hline USA一Seattle [47] & July 1978-December 1983 & 127 & 61.4 & $<43$ & Population & 174 & 71.3 & Population & 5 \\
\hline USA-New York City [48] & June 1979-February 1981 & 401 & 19.2 & $<30-70+$ & Hospital & 519 & 22.9 & Hospital & 5 \\
\hline Sweden—southern region [49] & 1979-1985 & 174 & 82.2 & $<45$ & Population & 459 & 71.7 & Population & 5 \\
\hline former Yugoslavia-Slovenia [50] & January 1980-September 1983 & 534 & 30.3 & $24-54$ & Hospital & 1989 & 23.9 & Hospital & 5 \\
\hline Italy_Pordenone [51] & January 1980-March 1983 & 368 & 4.1 & $27-79$ & Hospital & 373 & 5.9 & Hospital & 5 \\
\hline UK-London. Oxford [52] & September 1980-1984 & 1125 & 37.7 & $16-64$ & Hospital & 1125 & 38.8 & Hospital & 5 \\
\hline $\begin{array}{c}\text { Cancer and Steroid Hormone Study } \\
\text { USA-Atlanta. Connecticut, Detroit. } \\
\text { Iowa, New Mexico. San Francisco. } \\
\text { Seattle, Utach [53] }\end{array}$ & December 1980-December 1982 & 4341 & 57.7 & $20-54$ & Population & 4343 & 59.2 & Population & 5 \\
\hline Australia—New South Wales [54] & 1980-1982 & 141 & 48.2 & $25-64$ & Hospital & 279 & 42.3 & Hospital & 5 \\
\hline
\end{tabular}


Table 1. Cont

\begin{tabular}{|c|c|c|c|c|c|c|c|c|c|}
\hline $\begin{array}{c}\text { Study Name } \\
\text { Setting [Reference] }\end{array}$ & Recruitment Period & $\begin{array}{l}\text { Number of } \\
\text { Case Subjects }\end{array}$ & $\begin{array}{l}\text { Percent of } \\
\text { OCs Users }\end{array}$ & $\begin{array}{c}\text { Age Range } \\
\text { (Years) }\end{array}$ & $\begin{array}{l}\text { Source of } \\
\text { Cases }\end{array}$ & $\begin{array}{c}\text { Number of } \\
\text { Controls Subjects }\end{array}$ & $\begin{array}{l}\text { Percent of } \\
\text { OCs Users }\end{array}$ & $\begin{array}{l}\text { Source of } \\
\text { Controls }\end{array}$ & NOS Sc. \\
\hline $\begin{array}{l}\text { UK National Case-Control Study } \\
\text { UK-England and Scotland [55] }\end{array}$ & January 1982-December 1985 & 755 & 91.1 & $<35$ & Population & 755 & 89.3 & Clinic & 6 \\
\hline Costa Rica [56] & January 1982-March1984 & 155 & 37.4 & $20-49$ & Population & 748 & 42.9 & Population & 5 \\
\hline Australia_Adelaide [57] & April 1982-July 1984 & 395 & 48.9 & $20-69$ & Population & 386 & 48.7 & Population & 5 \\
\hline $\begin{array}{l}\text { WHO Collaborative Study of Neoplasia } \\
\text { and Steroid Contraceptives * [58] }\end{array}$ & November 1982-February 1986 & 2116 & 34.0 & $20-59$ & Hospital & 13072 & 33.9 & Hospital & 7 \\
\hline Italy_greater Milan area [60] & January 1983-December 1991 & 2309 & 16.1 & $22-59$ & Hospital & 1928 & 13.7 & Hospital & 5 \\
\hline USA_Seattle metropolitan area [61] & January 1983-April 1990 & 747 & 92.2 & $21-45$ & Population & 961 & 91.5 & Population & 6 \\
\hline Denmark [62] & March 1983-August 1984 & 1059 & 45.6 & $<59$ & Population & 990 & 47.0 & Population & 5 \\
\hline $\begin{array}{l}\text { USA-San Francisco-Oakland, Los } \\
\text { Angeles, Oahu [63] }\end{array}$ & April 1983-June 1987 & 590 & 35.1 & $20-55$ & Population & 945 & 37.1 & Population & 6 \\
\hline $\begin{array}{c}\text { Auckland Breast Cancer Study } \\
\text { New Zealand [64] }\end{array}$ & July 1983-June 1987 & 891 & 76.9 & $25-54$ & Population & 1861 & 82.5 & Population & 6 \\
\hline $\begin{array}{l}\text { France-Strasburg, Lyons, } \\
\text { Tours, Marseilles [66] }\end{array}$ & 1983-1987 & 464 & 51.1 & $20-55$ & Hospital & 542 & 43.5 & Hospital & 5 \\
\hline USA—Nassau and Suffolk County [67] & January 1984-December 1986 & 1420 & 25.9 & $20-79$ & Population & 1420 & 22.6 & Population & 5 \\
\hline Sweden and Norway [68] & May 1984-May 1985 & 422 & 77.3 & $20-49$ & Population & 527 & 70.4 & Population & 5 \\
\hline China-Shanghai [69] & June 1984-May 1985 & 534 & 18.5 & $20-69$ & Population & 534 & 17.8 & Population & 5 \\
\hline China-Tiajin [70] & January 1985-November 1986 & 300 & 34.7 & $20-55$ & Hospital & 300 & 32.7 & Population & 5 \\
\hline Spain—Girona [71] & July 1986-June 1993 & 330 & 11.5 & $<75$ & Population & 346 & 18.5 & Population & 6 \\
\hline $\begin{array}{l}\text { Netherlands Oral Contraceptives and } \\
\text { Breast Cancer Study The Netherlands [72] }\end{array}$ & October 1986-June 1989 & 918 & 85.4 & $20-54$ & Population & 918 & 85.2 & Population & 6 \\
\hline Singapore [73] & $1986-1988$ & 200 & 25.5 & $<40-70+$ & Hospital & 420 & 25.2 & Hospital & 5 \\
\hline Slovenia [74] & January 1988-December 1990 & 624 & 47.8 & $25-54$ & Population & 624 & 47.9 & Population & 6 \\
\hline USA-King County [75] & January 1988-June 1990 & 537 & 47.1 & $50-64$ & Population & 492 & 45.9 & Population & 6 \\
\hline $\begin{array}{c}\text { USA-Wisconsin, Maine, } \\
\text { Massachusetts (excluding Boston), } \\
\text { New Hampshire [76] }\end{array}$ & April 1988-December 1991 & 6751 & 35.5 & $<75$ & Population & 9311 & 37.0 & Population & 5 \\
\hline
\end{tabular}


Table 1. Cont.

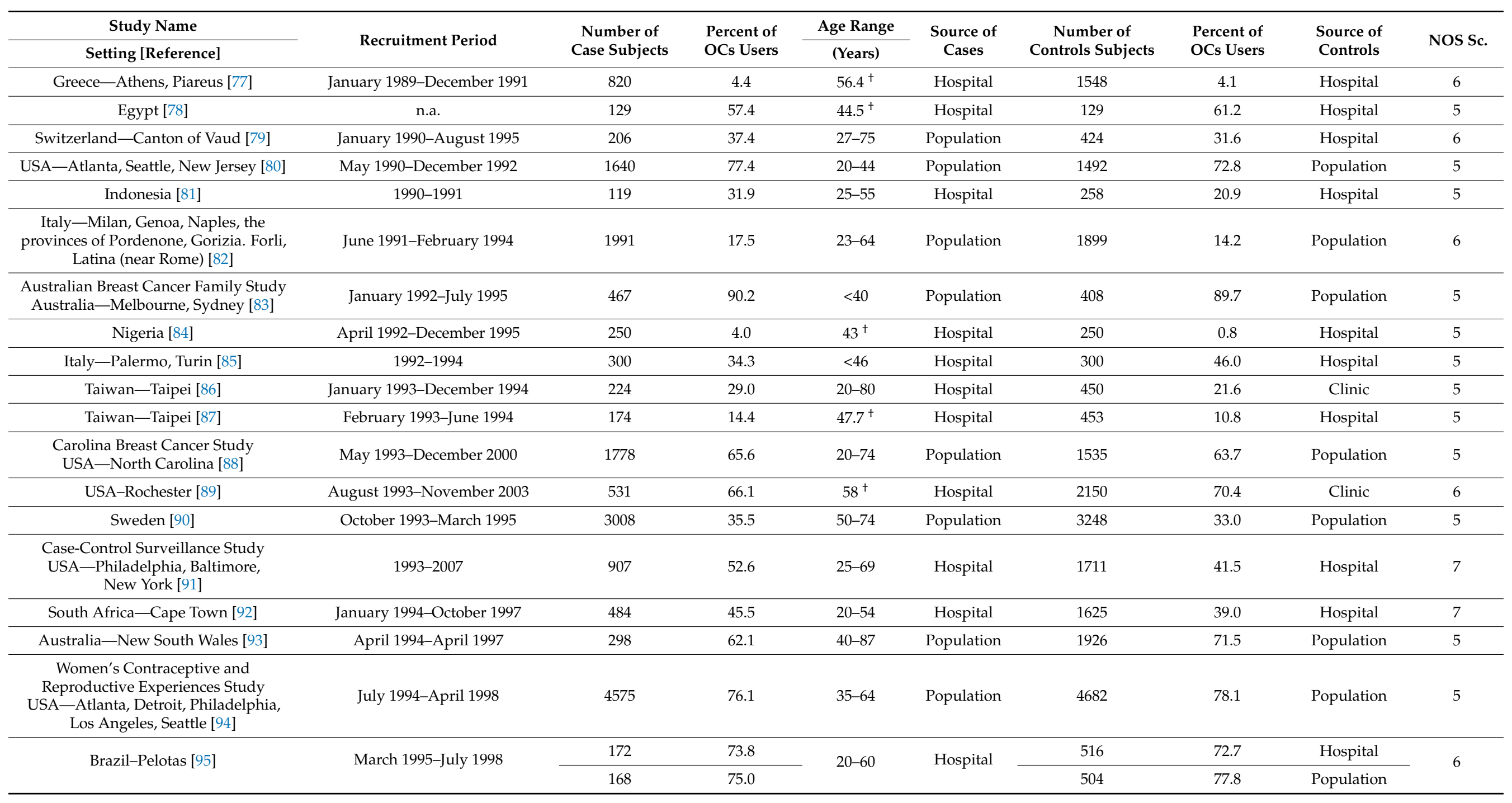


Table 1. Cont.

\begin{tabular}{|c|c|c|c|c|c|c|c|c|c|}
\hline $\begin{array}{c}\text { Study Name } \\
\text { Setting [Reference] }\end{array}$ & Recruitment Period & $\begin{array}{l}\text { Number of } \\
\text { Case Subjects }\end{array}$ & $\begin{array}{l}\text { Percent of } \\
\text { OCs Users }\end{array}$ & $\begin{array}{c}\text { Age Range } \\
\text { (Years) }\end{array}$ & $\begin{array}{l}\text { Source of } \\
\text { Cases }\end{array}$ & $\begin{array}{c}\text { Number of } \\
\text { Controls Subjects }\end{array}$ & $\begin{array}{l}\text { Percent of } \\
\text { OCs Users }\end{array}$ & $\begin{array}{l}\text { Source of } \\
\text { Controls }\end{array}$ & NOS Sc \\
\hline $\begin{array}{l}\text { Long Island Breast Cancer Study } \\
\text { Project USA-Long Island [96] }\end{array}$ & August 1996-July 1997 & 1475 & 43.2 & $20-98$ & Population & 1492 & 44.7 & Population & 6 \\
\hline Iran-Tehran [97] & April 1997-April 1998 & 286 & 2.4 & $24-81$ & Hospital & 249 & 3.6 & Hospital & 5 \\
\hline Turkey_Ankara [98] & January 1998-September 1999 & 504 & 23.6 & $49.4^{+}$ & Hospital & 610 & 16.9 & Hospital & 5 \\
\hline Cyprus [99] & January 1999-December 2005 & 1109 & 25.3 & $40-70$ & Population & 1177 & 25.0 & Population & 6 \\
\hline $\begin{array}{c}\text { British Women's Heart and Health } \\
\text { Cohort Study } \\
\text { UK-Nationwide [100] }\end{array}$ & April 1999-March 2001 & 225 & 25.4 & $60-79$ & Population & 4061 & 23.9 & Population & 5 \\
\hline $\begin{array}{c}\text { USA-Arizona, Colorado, Utah, } \\
\text { New Mexico [101] }\end{array}$ & October 1999-May 2004 & 2303 & 64.9 & $<64$ & Population & 2513 & 59.8 & Population & 7 \\
\hline Turkey-Istanbul [102] & January 2000-December 2006 & 1492 & 18.4 & $35-70$ & Hospital & 2167 & 27.8 & Clinic & 7 \\
\hline Iran-Bandar Abbas [103] & April 2000-March 2002 & 168 & 18.5 & $27-92$ & Population & 504 & 20.0 & Population & 6 \\
\hline Malaysia-Kelantan [104] & July 2000-June 2001 & 147 & 36.1 & $26-70$ & Hospital & 147 & 24.5 & Hospital & 5 \\
\hline Turkey-Istanbul [105] & September 2002-October 2003 & 405 & 23.0 & $28-72$ & Hospital & 1050 & 14.7 & Hospital & 7 \\
\hline Germany [107] & 2004-2005 & 3593 & 69.8 & n.a. & Population & 9098 & 79.8 & Population & 5 \\
\hline Iran-Teheran [108] & 2004 & 300 & 59.0 & $24-84$ & Hospital & 303 & 49.2 & Hospital & 6 \\
\hline Pakistan-Islamabad [109] & January 2005-July 2005 & 132 & 18.2 & $42.4^{\dagger}$ & Hospital & 145 & 9.0 & Hospital & 6 \\
\hline
\end{tabular}

* Participating countries: Australia. Chile. the People's Republic of China. Colombia. the German Democratic Republic (GDR). Israel. Kenya. Mexico. the Philippines. and Thailand ${ }^{+}$mean. 


\subsection{Summary Analysis}

Of the 79 case-control studies, 17 reported a significant positive relationship for OC use and risk of BrCa $[40,46,49,50,60,66,68,80-82,84,91,92,101,104,105,109]$, and a further 26 reported a positive but non-significant association $[31,38,45,54,55,57,58,61,67,69$, $70,72,73,75,77-79,83,86-88,90,99,100,108]$. In contrast, nine studies reported a significant inverse association $[33,36,41,64,76,85,93,98,102]$, and 27 reported a non-significant inverse association $[32,34,35,37,39,42-45,47,48,51-53,56,59,62,63,65,71,74,89,94,96,97,103,106,107]$. Tessaro et al. [95] found a small increase in BrCa risk in a study using the control group of population (OR, 1.06; 95\% CI, 0.72-1.57), and reduced risk with a control group of hospital patients (OR, 0.86; 95\% CI, 0.57-1.29).

The present meta-analysis based on reanalysis and summary analysis of available data showed no difference in the association between OC ever-use and breast cancer risk, compared with never-use (crude OR (cOR), 1.01; 95\% CI, 0.95-1.07; $p=0.69$ ) (Figure 2). The Begg-Mazumdar and Egger tests showed no publication bias $(p>0.05)$. In addition, we performed separate analysis for assessment of relationship between risk of breast cancer and use of older oral contraceptives formulations containing higher doses of estrogen and oral contraceptives using low-estrogen formulations, based on the periods of recruitment to the study. Figure 3 (1966-1985) and Figure 4 (1986-2010) showed that in a studied subgroup, the overall estimates of $\mathrm{BrCa}$ risk slightly oscillated around the unit: $\mathrm{cOR}=0.99$ (95\% CI, 0.93-1.05; $p=0.71$ ) and $\mathrm{cOR}=1.04$ (95\% CI, 0.94-1.53; $p=0.41)$, respectively. Similar results were obtained for the matched control groups in the analyzed subgroups (Figures 2 and 3).

Our analysis was restricted to premenopausal or women younger than 50 years and showed a marginal, non-significant increase in risk of breast cancer with ever use of OCs (cOR, 1.06; 95\% CI, 0.92-1.22; $p=0.44$ ), compared with controls that had never used OCs (Figure 5). Analysis of risk among postmenopausal or women over age 50 ever using OCs also did not show difference (cOR, 0.99; (95\% CI, 0.89-1.10; $p=0.90)$ (Figure 6). Moreover, we indicated a small decrease in risk of developing breast cancer in OC users who began before age 25 years (cOR, 0.91; 95\% CI, 0.83-1.00; $p=0,05$ ) (Figure 7).

As shown in Figure 8, the use of OC by nulliparous women did not affect breast cancer risk (cOR, 1.02; 95\% CI, 0.82-1.26: $p=0.85$ ), although the use of pills before a first full-term pregnancy has a significant effect on increased risk of breast cancer (cOR, 1.14; 95\% CI, $1.01-1.28 ; p=0.04$ ), compared with never-use (Figure 9). Furthermore, OC use longer than 5 years leads to a slight, but significant increase of breast cancer risk (cOR, 1.09; 95\% CI, 1.01-1.18; $p=0.02$ ) (Figure 10). 


\begin{tabular}{|c|c|c|c|c|}
\hline \multirow[b]{2}{*}{$\begin{array}{l}\begin{array}{l}\text { References } \\
\text { First author }\end{array} \\
\end{array}$} & \multicolumn{2}{|c|}{ Use of oral contraceptives } & \multirow[b]{2}{*}{$(95 \% \mathrm{C})$} & \\
\hline & $\frac{\text { Kver }}{\text { Cases/Controls }}$ & $\frac{\text { Never }}{\text { Cases/Controls }}$ & & \\
\hline Adcbamowo [84] & $10 / 2$ & $240 / 248$ & $5.17(1.12-23.83)$ & 0.0352 \\
\hline Afthuis $[80]$ & 1269/1086 & $371 / 406$ & $1.28(1.09-1.50)$ & 0.0030 \\
\hline Beji [105] & 93/154 & $312 / 896$ & $1.73(1.30-2.31)$ & 0.0002 \\
\hline Bustan [8]] & $38 / 54$ & $81 / 204$ & $1.77(1.092 .89)$ & 0.0216 \\
\hline Chie $[87]$ & $25 / 47$ & 149/406 & $1.45(0.86-2.41)$ & 0.1620 \\
\hline Clavel [66] & $237 / 236$ & $227 / 306$ & $1.35(1.06-1.74)$ & 0.0171 \\
\hline Croghan [89] & 351/1514 & $180 / 636$ & $0.82(0.67-1.00)$ & 0.0531 \\
\hline Dinger [107] & $2508 / 7271$ & $1079 / 1805$ & $0.58(0.53-0.63)$ & 0.0000 \\
\hline Fbrahimi [97] & 79 & $279 / 210$ & $0.67(0.25-1.82)$ & 0.4321 \\
\hline Elkery [54] & $67 / 18$ & 73/161 & $1.25(0.83-1.88)$ & 0.2797 \\
\hline Rwert: [162] & $479 / 45 \mathrm{~s}$ & $576 / 525$ & $0.95(0.80-1.1 .3)$ & 0.5904 \\
\hline Faheern [109] & $24 / 13$ & $108 / 132$ & $2.26(1.10-1.61)$ & 0.0270 \\
\hline Corres [46] & $60 / 82$ & $233 / 484$ & $1.52(1.05-2.20)$ & 0.0257 \\
\hline Hadjisavvas $[\mathscr{Y}]]$ & $280 / 294$ & 823/A79 & $1.02(0.84-1.23)$ & 0.8599 \\
\hline Hall [s8] & 1160/6ss & $612 / 557$ & $1.09(0.95-1.26)$ & 0.2344 \\
\hline I larris [43] & 36/189 & $73 / 279$ & $0.73(0.47-1.13)$ & 0.1571 \\
\hline I larris $[48]$ & 77n19 & $324 / 400$ & $0.80(0.58-1.10)$ & 0.1715 \\
\hline Hennekens [31] & $273 / 2641$ & $716 / 7260$ & $1.05(0.91-1.21)$ & 0.5290 \\
\hline Janerich [40] & $153 / 233$ & $120 / 264$ & $1.44(1.07-1.94)$ & 0.0152 \\
\hline Jick [47] & $78 / 124$ & $28 / 29$ & $0.65(0.36-1.18)$ & 0.1557 \\
\hline Kamarudin [106] & $62 / 78$ & $118 / 105$ & $0.71(0.46-1.08)$ & 0.1100 \\
\hline Kishk [78] & $55 / 50$ & $74 / 79$ & $1.17(0.71-1.93)$ & 0.5264 \\
\hline Kuru [98] & 119/103 & $30 / 12$ & $0.46(0.23-0.95)$ & 0.0355 \\
\hline La Vecchia [82] & $348 / 270$ & $1643 / 1629$ & $1.28(1.07-1.52)$ & 0.0055 \\
\hline I.awlor [1000] & $57 / 971$ & 168/3090 & $1.08(0.79-1.47)$ & 0.6266 \\
\hline J.ee [56] & $58 / 321$ & $97 / 427$ & $0.80(0.56-1.14)$ & 0.2077 \\
\hline Lee $[73]$ & $51 / 106$ & $148 / 310$ & $1.01(0.68-1.48)$ & 0.9687 \\
\hline Lees [36] & $105 / 239$ & $190 / 268$ & $0.62\left(\begin{array}{lll}0.46 & 0.83)\end{array}\right)$ & 0.0015 \\
\hline I.evi $[79]$ & $77 / 34$ & $129 / 290$ & $1.29(0.91-1.83)$ & 0.1501 \\
\hline Lipworth [77] & $36 / 63$ & $784 / 1485$ & $1.08(0.71-1.64)$ & 0.7109 \\
\hline Lubin [41] & $151 / 229$ & $426 / 477$ & $0.51(0.41-0.65)$ & 0.0000 \\
\hline I. und [68:] & $326 / 371$ & $96 / 156$ & $1.43(1.06-1.92)$ & 0.0178 \\
\hline Magrusson [90] & 898/1889 & 1733//1938 & $1.13(1.01-1.26)$ & 0.0347 \\
\hline Mahouri [103] & 31/101 & 137/403 & $0.90(0.58-1.41)$ & 0.6539 \\
\hline Marchbanka [99] & $3497 / 3655$ & (1032P\$P & $0.91(0.82-1.100)$ & 0.0554 \\
\hline MeCredic [83] & $418 / 366$ & $46 / 42$ & $1.04(0.67-1.62)$ & 0.8524 \\
\hline Merherson [52] & 424/436 & 701/689 & $0.96(0.81-1.13)$ & 0.6026 \\
\hline Newcomb [76] & $2262 / 3447$ & $4489 / 5864$ & $0.86(0.80-0.92)$ & 0.00000 \\
\hline Nonsa'adah [104] & $53 / 36$ & $94 / 11$ & $1.74(1.05-2.88)$ & 0.03317 \\
\hline Olssun [49] & $143 / 229$ & 31/130 & $1.82(1.18-2.83)$ & 0.0072 \\
\hline Ormen [102] & $275 / 602$ & 121717565 & $0.59(0.50-0.69)$ & 0.0000 \\
\hline Paffenbarger [35] & 536/10002 & $1322 / 2366$ & $0.96(0.85-1.08)$ & 0.4932 \\
\hline Palmer [45] & $161 / 275$ & $358 / 733$ & $1.20(0.95-1.51)$ & 0.1256 \\
\hline Paal [64] & $685 / 1536$ & $206 / 325$ & $0.70\left(\begin{array}{lll}0.58 & 0.86\end{array}\right)$ & 0.0005 \\
\hline Pike [38] & 135/218 & $28 / 52$ & $1.15(0.69-1.91)$ & 0.5889 \\
\hline Price [93] & 185/1378 & $96 / 508$ & $0.71(0.51-0.93)$ & 0.0120 \\
\hline Primic-Žakcli [74] & 298/299 & $326 / 325$ & $0.99(0.80-1.24)$ & 0.9548 \\
\hline Ravnibar [37] & $30 / 65$ & $160 / 315$ & $0.91(0.57-1.46)$ & 0.6912 \\
\hline Ravnihar [50] & 162/167 & $372 / 1522$ & $1.12(1.15-1.75)$ & 0.0012 \\
\hline Rohan [57] & 193/188 & $201 / 198$ & $1.01(0.76-1.34)$ & 0.9376 \\
\hline Rookus |72] & $784 / 782$ & $134 / 136$ & $1.02(0.79-1.32)$ & 0.8951 \\
\hline Rosenbery [59] & $260 / 544$ & $347 / 670$ & $0.92(0.76-1.12)$ & 0.4232 \\
\hline Rosenberg [M] & 1028/1361 & $2439 / 3069$ & $0.95(0.86-1.05)$ & 0.3037 \\
\hline Roscrnberg [191] & $477 / 710$ & 430/1001 & $1.56(1.33-1.84)$ & 0.0000 \\
\hline Kossing, 175] & $253 / 226$ & $239 / 266$ & $1.05(0.82-1.34)$ & 0.7050 \\
\hline Sartwell [39] & $22 / 34$ & $262 / 333$ & $0.22(0.47-1.44)$ & 0.4939 \\
\hline Schildkraut | & 66/552 & 92/5588 & $0.76(0.55-1.07)$ & 0.1176 \\
\hline Shanlakumar $l^{\circ}$ & $637 / 667$ & 838/825 & $0.94(0.81-1.09)$ & 0.4047 \\
\hline Shapim [92] & $2201 / 633$ & $264 \times 1942$ & $1.31(1.106-1.60)$ & 0.0106 \\
\hline Stanford [39] & $481 / 534$ & $1540 / 1667$ & $0.98(0.85-1.12)$ & 0.7259 \\
\hline Sweonoy [101] & $1494 / 1502$ & 809/1011 & $1.24(1.11-1.40)$ & 0.0003 \\
\hline Talaminin [5]] & $15 / 22$ & 353/351 & $0.68(0.35-1.33)$ & 0.2575 \\
\hline Tavani [60] & $371 / 265$ & $1938 / 1663$ & $1.20(1.01-1.43)$ & 0.0352 \\
\hline Tessara [95|" & $127 / 45$ & 375//41 & $1.06(0.72-1.57)$ & 0.7662 \\
\hline Tessaro [955|t & $126 / 42$ & 392/112 & $0.86(0.57-1.29)$ & 0.4584 \\
\hline Traina [85] & $103 / 13$ & 197/162 & (14-0.85) & 0.0037 \\
\hline UK NCCS 15 & $688 / 675$ & $67 / 80$ & 86-1.1.71) & 0.2596 \\
\hline Unsin [65] & $618 / 626$ & 124/116 & $0.92(0.70-1.22)$ & 0.5728 \\
\hline Unsin [63] & $207 / 35$ & $383 / 594$ & $0.91(0.71-1.13)$ & 0.4149 \\
\hline Vessey [32] & 537/: & $639 / 622$ & $94(0.80-1.11)$ & 0.4821 \\
\hline Viladiu [71] & 38/54 & $292 / 292$ & $0.70(0.45-1.10)$ & 0.1222 \\
\hline Warg $1700 \mid$ & 1049P5 & $166 / 202$ & $1.099(0.78-1.53)$ & 0.69013 \\
\hline Weinstein & & $789 / / 222$ & (298-1.46) & 0.0726 \\
\hline Whate [61] & 6899879 & $58 / 82$ & $1.11(0.78-1.57)$ & 0.5659 \\
\hline Wilostudy I & 7201/4427 & 1396/864t & $1.01(0.91-1.11)$ & 0.8852 \\
\hline Wingo [53] & $251 / 2 / 2569$ & 1кз8 & $0.94(0.86-1.02)$ & 0.1514 \\
\hline Wynder [33] & 721288 & 446/1313 & $0.74(0.56-0.97)$ & 0.0317 \\
\hline Yang $\left.\right|^{8}$ & $65 / 977$ & 179,353 & $1.32(0.92-1.90)$ & 0.1312 \\
\hline Yavari [1] & $177 / 149$ & $123 / 154$ & $1.49(1.05-2.05)$ & 0.0157 \\
\hline & & $435 / 439$ & & 0.7509 \\
\hline & & $770 / 70,278$ & $1.01(0.95-1.07)$ & 0.6876 \\
\hline
\end{tabular}

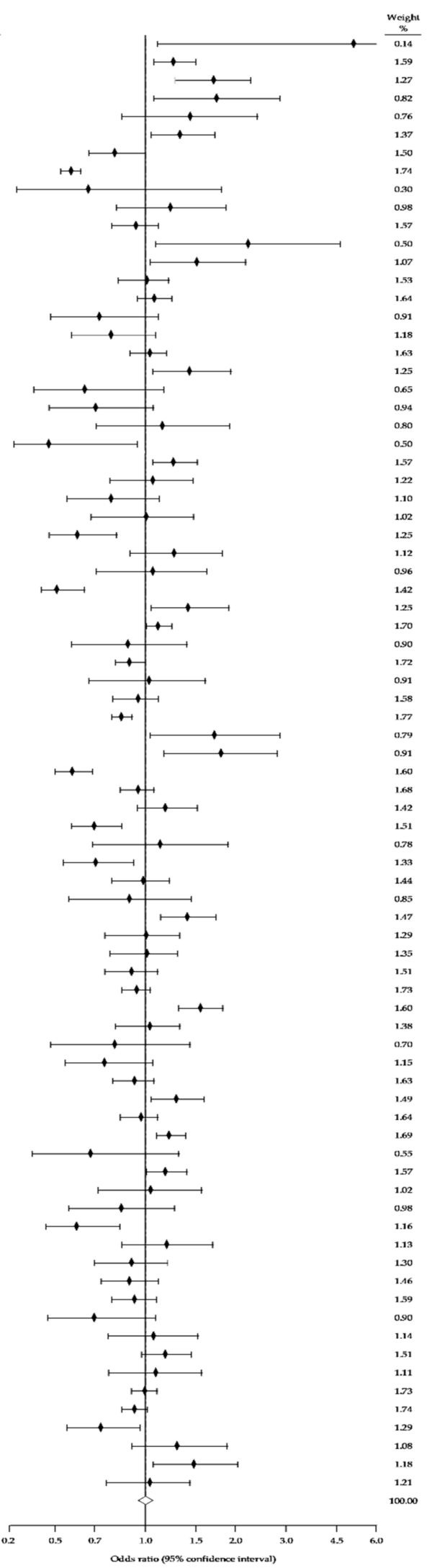

Figure 2. Forest plot and summary odds ratios on the association between risk of breast cancer and ever use of oral contraceptives: case-control studies conducted between 1960-2008, in alphabetical order. UK NCCS-UK National Case-control Study, WHO-World Health Organization, * neighborhood controls, ${ }^{+}$hospital controls. 


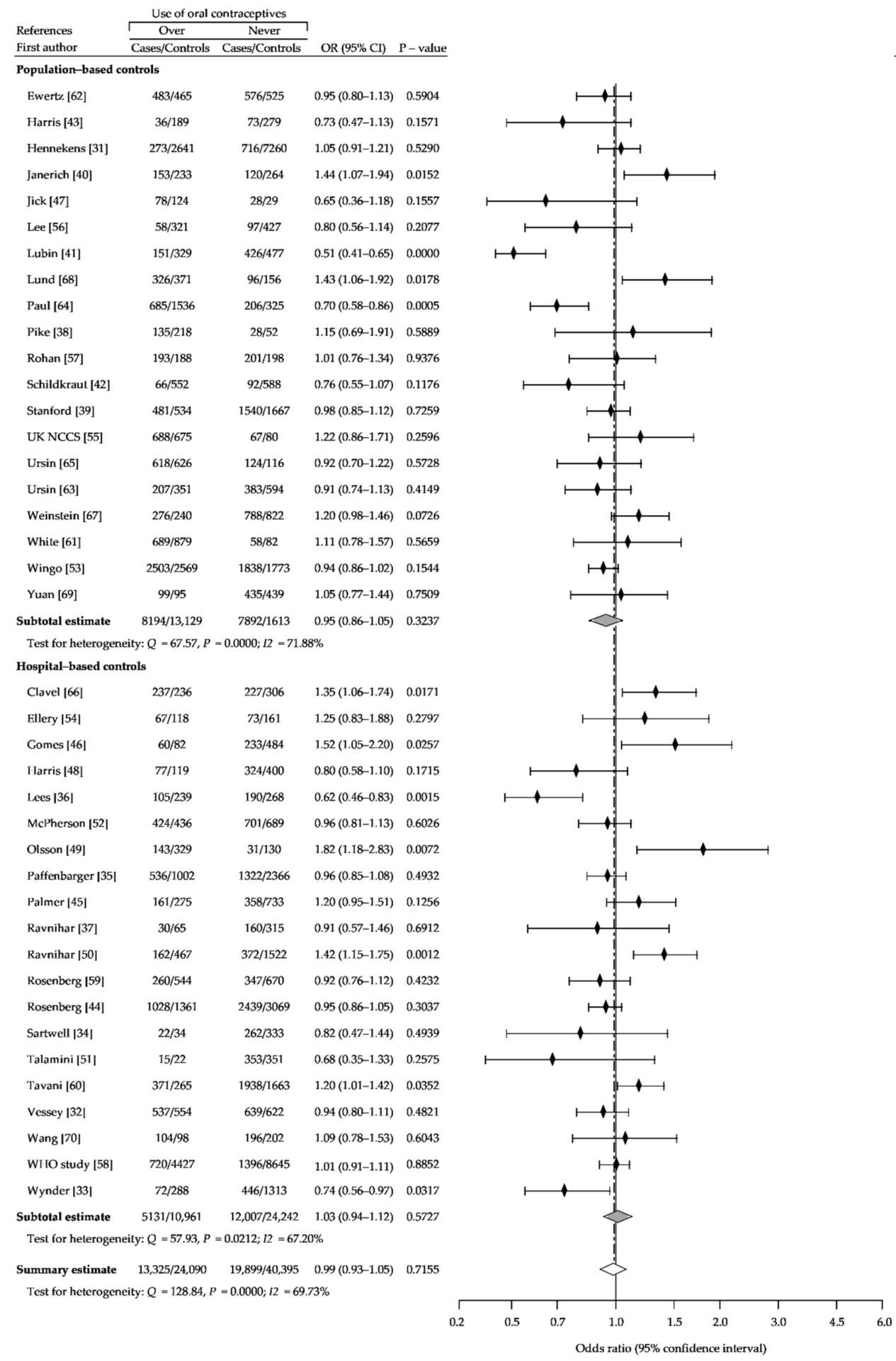

Weight

(\%)

6.51

3.13

6.92

4.71

2.09

3.97

5.62

4.74

6.17

2.60

4.93

4.20

6.99

4.14

4.99

5.90

6.14

4.03

7.68

4.52

100.00

5.24

Figure 3. Forest plot and summary odds ratios on the association between risk of breast cancer and ever use of oral contraceptives: case-control studies of the period of recruitment into the study before 1986, in alphabetical order. UK NCCS_UK National Case-control Study, WHO_World Health Organization. 


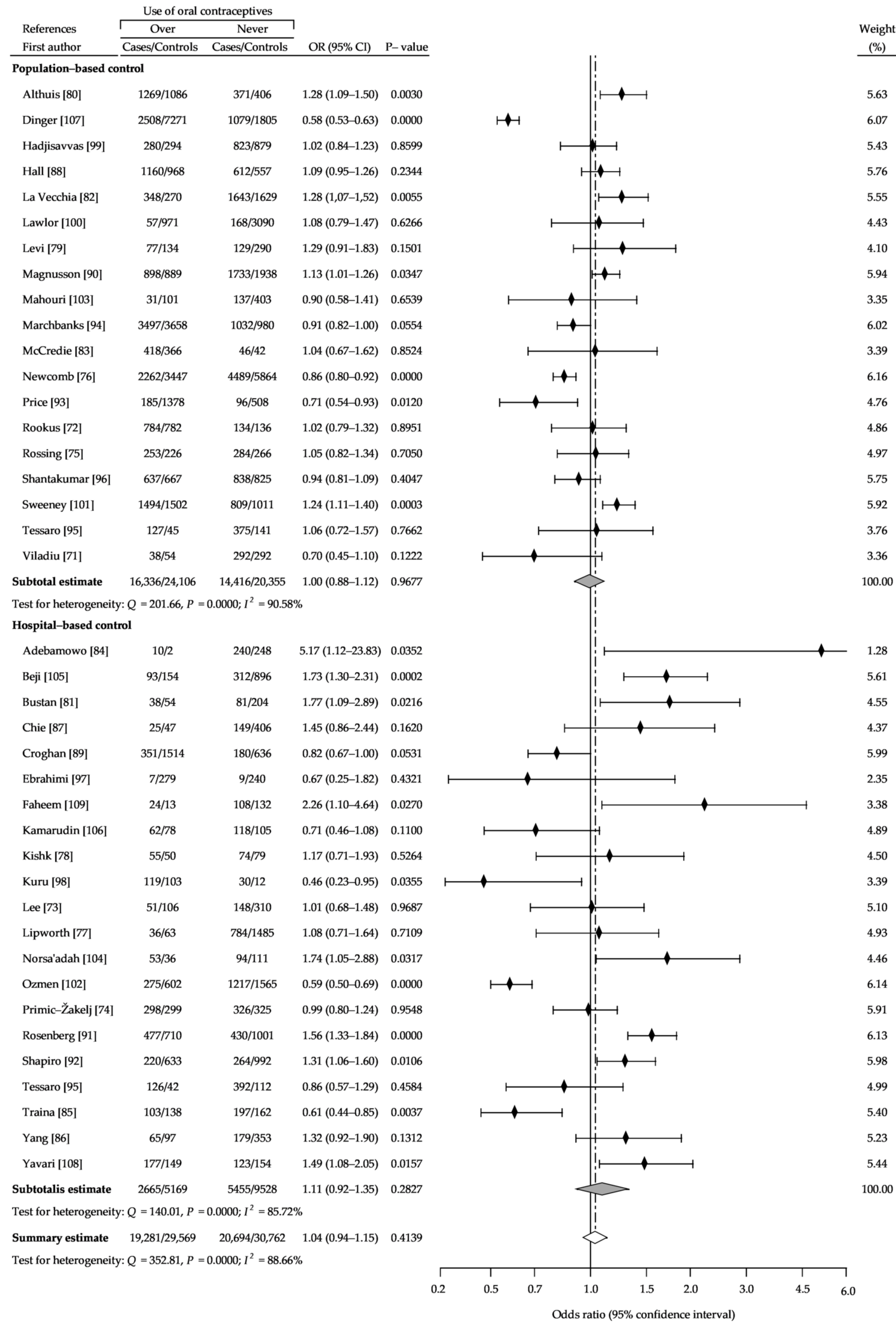

Figure 4. Forest plot and summary odds ratios on the association between risk of breast cancer and ever use of oral contraceptives: case-control studies of the period of recruitment into the study after 1986, in alphabetical order. 


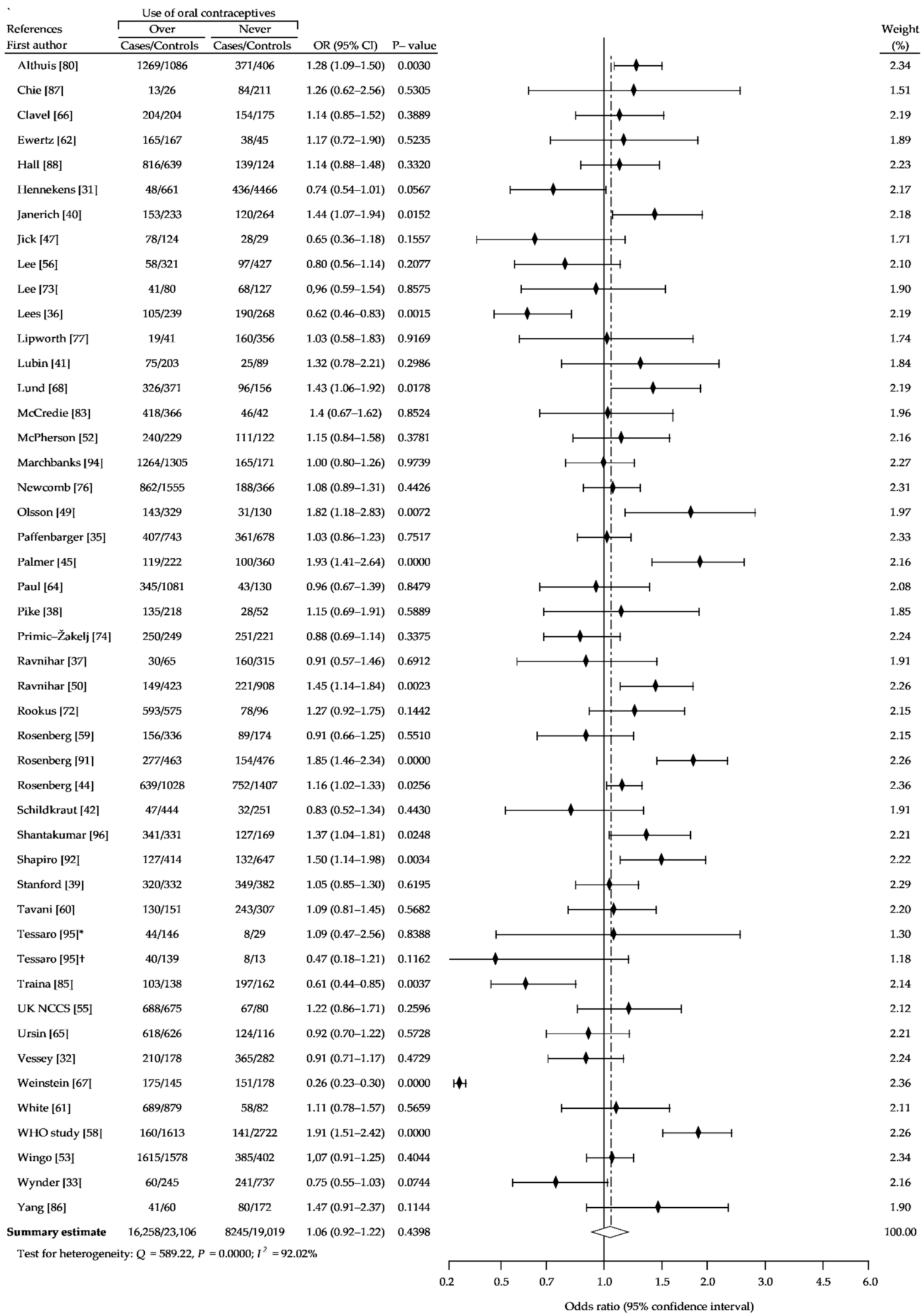

Figure 5. Forest plot and summary odds ratios on the association between risk of breast cancer and ever use of oral contraceptives in premenopausal women or women younger than 50 years: case-control studies in alphabetical order. UK NCCS-UK National Case-control Study, WHO-World Health Organization, ${ }^{*}$ neighborhood controls, ${ }^{\dagger}$ hospital controls. 


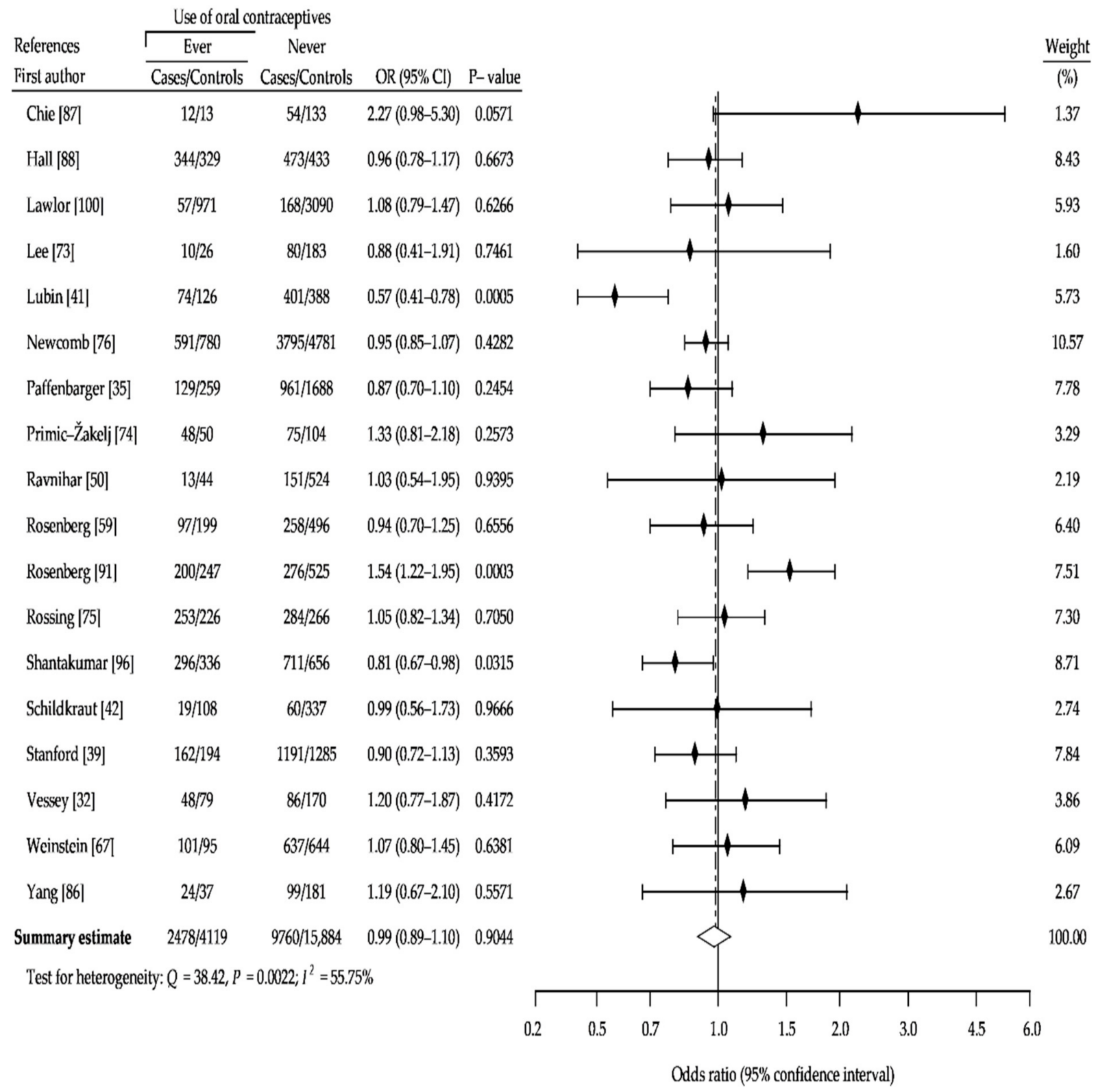

Figure 6. Forest plot and summary odds ratios on the association between risk of breast cancer and ever use of oral contraceptives in postmenopausal women or women older than 50 years: case-control studies in alphabetical order. 


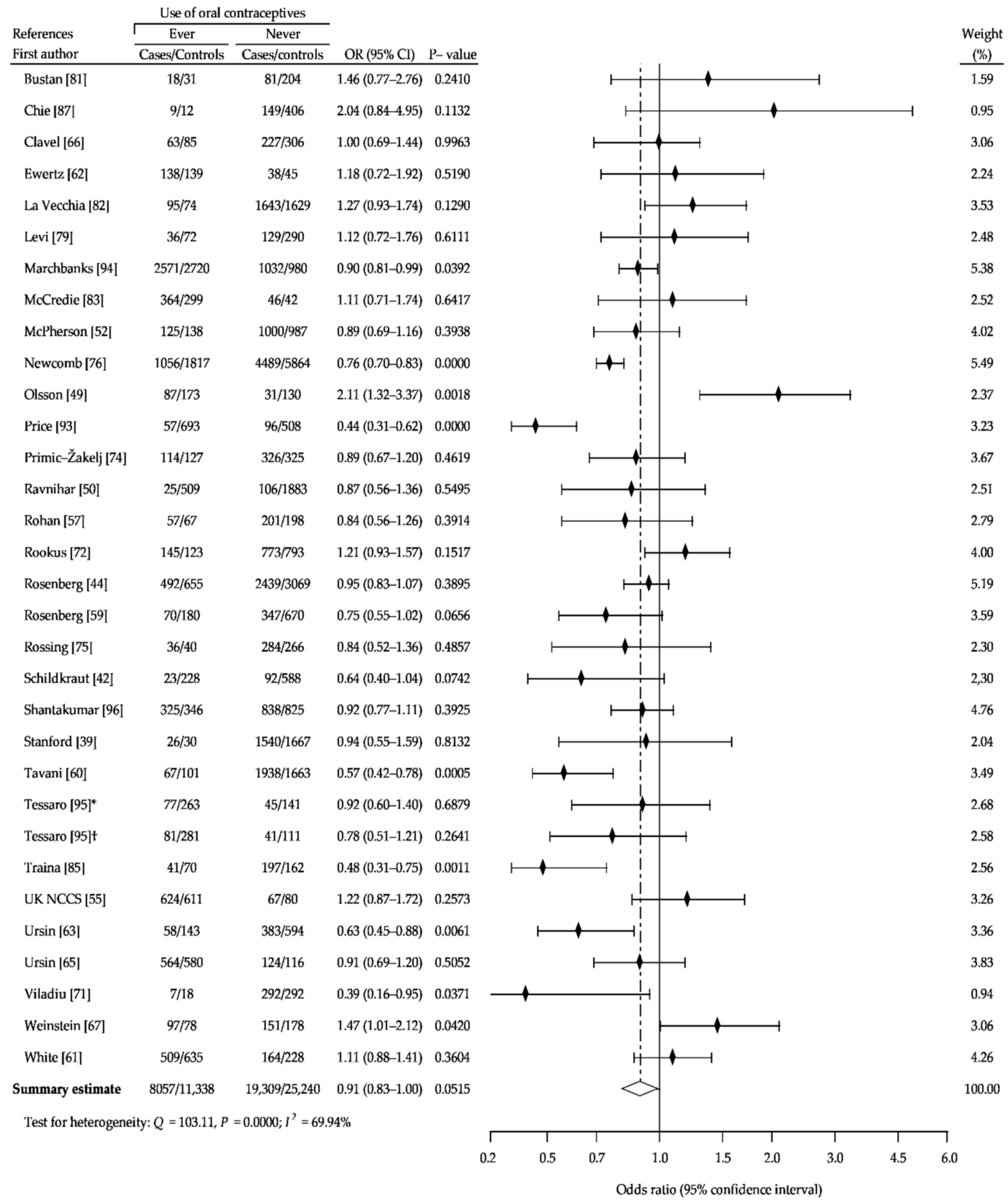

Figure 7. Forest plot and summary odds ratios on the association between risk of breast cancer and ever use of oral contraceptives in women under 25 years old: case-control studies in alphabetical order. UK NCCS-UK National Casecontrol Study, ${ }^{*}$ neighborhood controls, ${ }^{\dagger}$ hospital controls. 


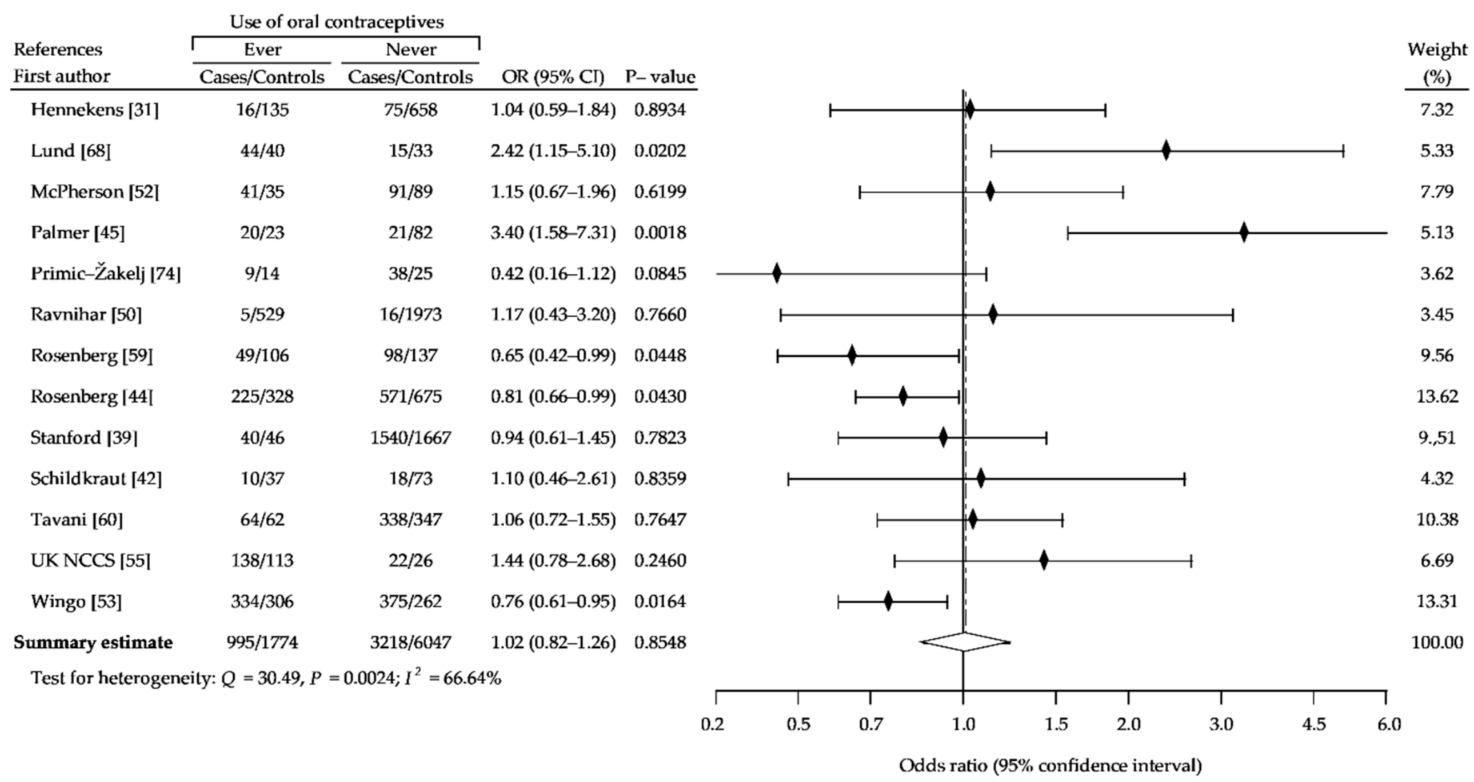

Figure 8. Forest plot and summary odds ratios on the association between risk of breast cancer and ever use of oral contraceptives use in nulliparous women: case-control studies in alphabetical order. UK NCCS—UK National Case-control Study.

\begin{tabular}{|c|c|c|c|c|}
\hline \multirow{2}{*}{$\begin{array}{l}\text { References } \\
\text { First author }\end{array}$} & \multicolumn{2}{|c|}{ Use of oral contraceptives } & \multirow{2}{*}{ OR $(95 \% \mathrm{Cl})$} & \multirow{2}{*}{$\mathrm{P}-$ value } \\
\hline & $\begin{array}{c}\text { Ever } \\
\end{array}$ & $\begin{array}{c}\text { Never } \\
\end{array}$ & & \\
\hline Chie [87] & $3 / 5$ & $149 / 406$ & $1.63(0.39-6.93)$ & 0.5045 \\
\hline Clavel [66] & $39 / 33$ & $179 / 250$ & $1.65(1.00-2.73)$ & 0.0503 \\
\hline Ellery [54] & $4 / 11$ & $63 / 107$ & $0.62(0.19-2.02)$ & 0.4258 \\
\hline Ewertz $|62|$ & $86 / 72$ & $38 / 45$ & $1.41(0.83-2.41)$ & 0.2026 \\
\hline Hennekens [31] & $33 / 328$ & $636 / 6241$ & $0.99(0.68-1.43)$ & 0.9455 \\
\hline Jick [47] & $28 / 44$ & $48 / 75$ & $0.99(0.55-1.81)$ & 0.9851 \\
\hline La Vecchia [82] & $90 / 51$ & $1643 / 1629$ & $1.75(1.23-2.48)$ & 0.0017 \\
\hline Lee [73] & $4 / 4$ & $87 / 166$ & $1.91(0.47-7.82)$ & 0.3691 \\
\hline Levi [79] & $23 / 47$ & $129 / 290$ & $1.10(0.64-1.89)$ & 0.7292 \\
\hline Lipworth [77] & $8 / 19$ & $160 / 356$ & $0.94(0.40-2.19)$ & 0.8800 \\
\hline Lund [68] & $143 / 149$ & $81 / 123$ & $1.46(1.01-2.09)$ & 0.0416 \\
\hline McCredie [83] & $269 / 208$ & $74 / 62$ & $1.08(0.74-1.59)$ & 0.6813 \\
\hline McPherson [52] & $137 / 94$ & 988/1031 & $1.52(1.15-2.01)$ & 0.0030 \\
\hline Newcomb [74] & $599 / 986$ & $4489 / 5864$ & $0.79(0.71-0.88)$ & 0.0000 \\
\hline Paffenbarger [35] & $33 / 29$ & $574 / 446$ & $0.88(0.53-1,48)$ & 0.6388 \\
\hline Palmer [45] & $32 / 48$ & $79 / 298$ & $2.51(1.51-4.19)$ & 0.0004 \\
\hline Pike [36] & $84 / 129$ & $79 / 141$ & $1.16(0.79-1.71)$ & 0.4489 \\
\hline Primic-Žakelj |74] & $33 / 37$ & $288 / 300$ & $0.93(0.57-1.53)$ & 0.7714 \\
\hline Ravnihar [49] & $7 / 527$ & $12 / 1977$ & $2.19(0.86-5.59)$ & 0.1014 \\
\hline Rohan [57] & $10 / 8$ & 201/198 & $1.23(0.48-3.18)$ & 0.6677 \\
\hline Rosenberg [44] & $286 / 263$ & $1866 / 2389$ & $1.39(1.17-1.66)$ & 0.0003 \\
\hline Schildkraut [42] & $19 / 130$ & $92 / 588$ & $0.93(0.55-1.59)$ & 0.8007 \\
\hline Sweeney [101] & $464 / 513$ & $809 / 1011$ & $1.13(0.97-1.32)$ & 0.1236 \\
\hline Tavani [60] & $56 / 44$ & $1938 / 1663$ & $1.09(0.73-1.63)$ & 0.6661 \\
\hline Traina [85] & $51 / 78$ & $197 / 162$ & $0.54(0.36-0.81)$ & 0.0030 \\
\hline UK NCCS [55] & $348 / 357$ & $247 / 259$ & $1.02(0.81-1.28)$ & 0.8509 \\
\hline Ursin [63] & $59 / 121$ & $383 / 594$ & $0.76(0.54-1.06)$ & 0.1038 \\
\hline Ursin [65] & $384 / 378$ & $124 / 16$ & $0.95(0.71-1.27)$ & 0.7309 \\
\hline Weinstein [67] & $70 / 52$ & $151 / 178$ & $1.59(1.04-2.41)$ & 0.0309 \\
\hline White [61] & $434 / 540$ & $164 / 228$ & $1.12(0.88-1.42)$ & 0.3591 \\
\hline Summary estimate & $3836 / 5305$ & $15,968 / 27,193$ & $1.14(1.01-1.28)$ & 0.0364 \\
\hline
\end{tabular}

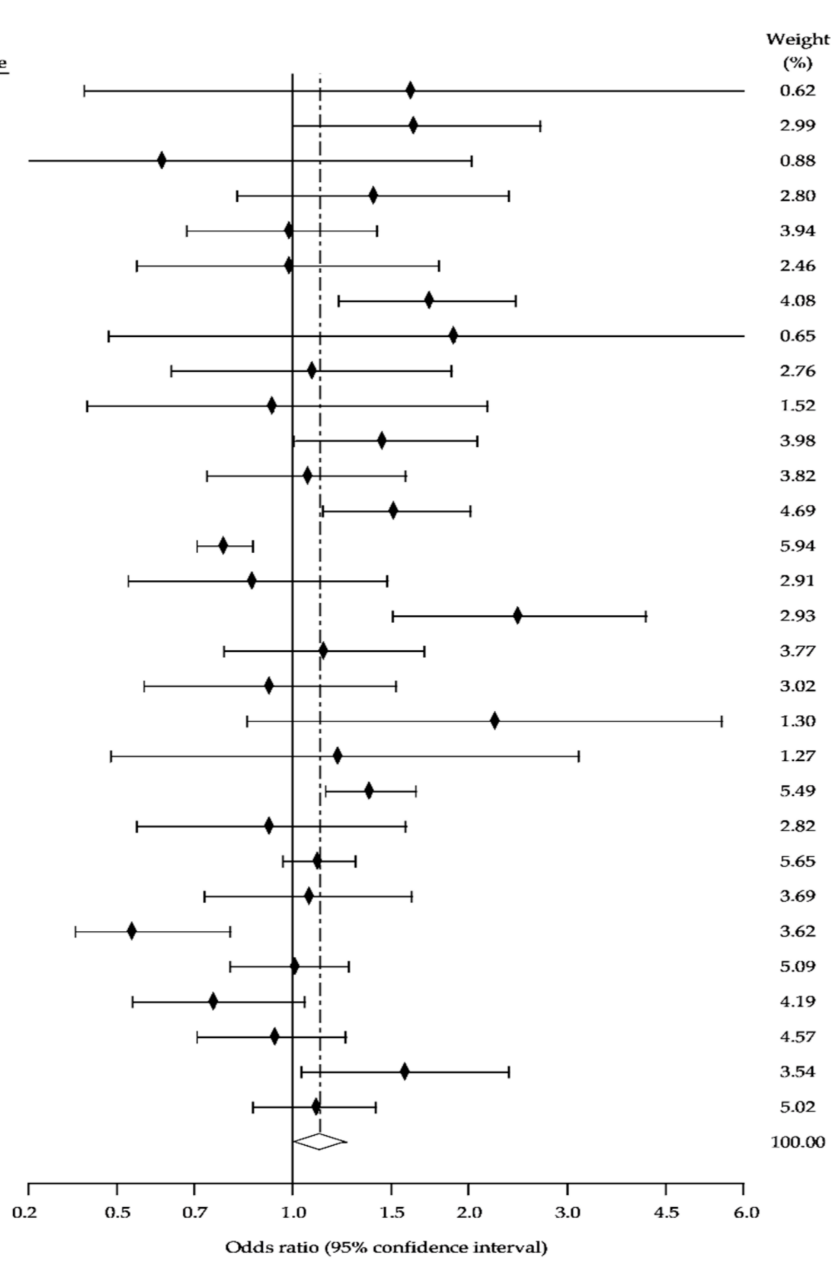

Figure 9. Forest plot and summary odds ratios on the association between risk of breast cancer and ever use of oral contraceptives use before first pregnancy: case-control studies in alphabetical order. UK NCCS-UK National Case-control Study. 


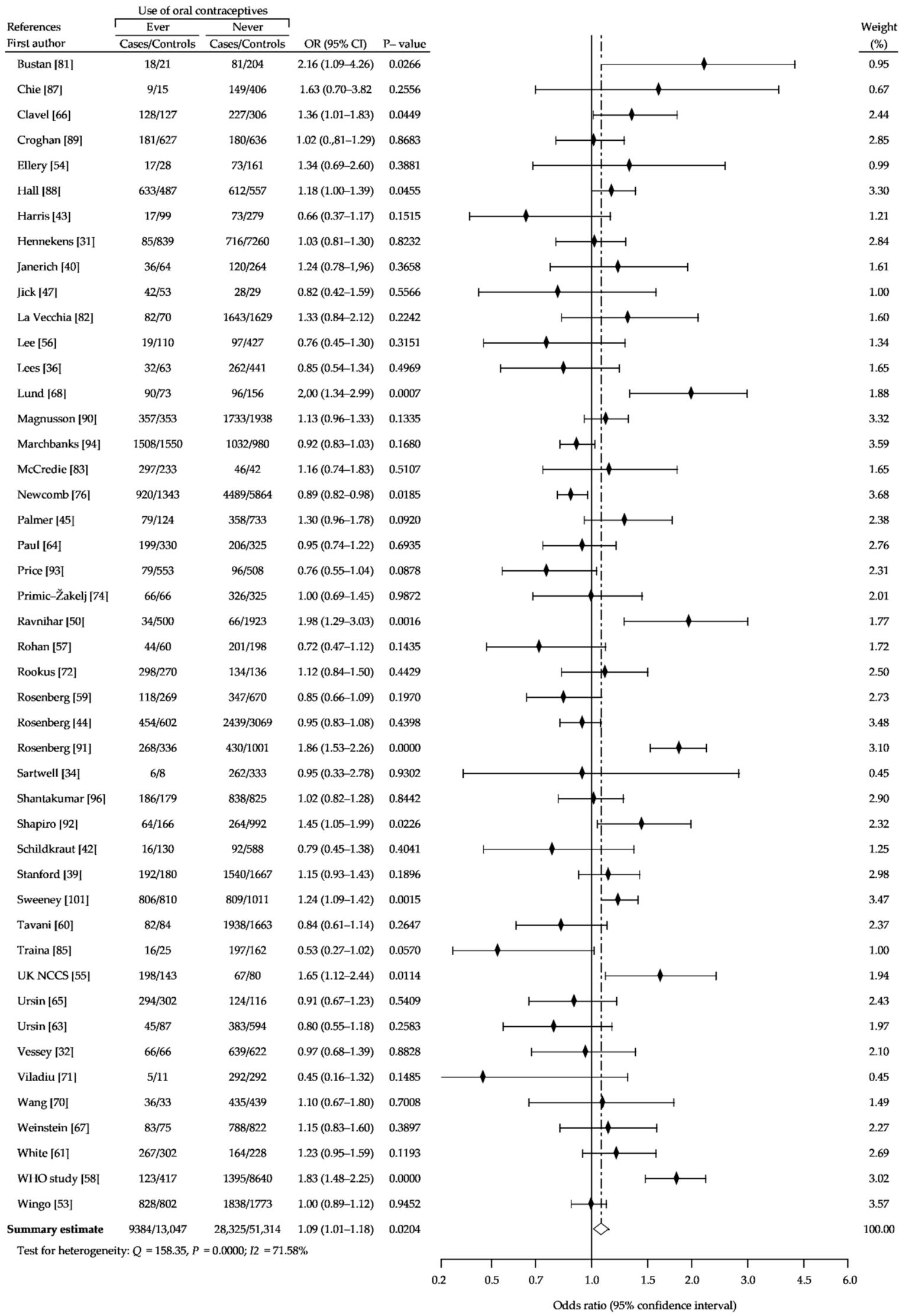

Figure 10. Forest plot and summary odds ratios on the association between risk of breast cancer and ever use of oral contraceptives longer than 5 years: case-control studies in alphabetical order. UK NCCS-UK National Case-control Study, WHO-World Health Organization. 


\section{Discussion}

Despite numerous epidemiological studies that have attempted to assess the risk of breast cancer among oral contraception users, the effect of oral contraceptive use on the developing breast cancer remains controversial. Several meta-analyses and a very large pooled analysis have addressed this issue. Romieu et al. [110], in meta-analysis based on 27 case-control studies published in 1966-1989, showed a marginally, non-significant increase in the risk of breast cancer among women ever using oral contraceptives [Relative Risk (RR), 1.06; 95\% CI, 0.98-1.14)). Delgado-Rodriguez et al. [20], in their meta-analysis including 40 case-control and 7 cohort studies published between 1966-1990, found that OC use was associated with slightly, but significantly, increased BrCa risk (RR, 1.06; 95\% CI, 1.02-1.10). In the same year, Thomas [111] presented a meta-analysis of data from 17 casecontrol studies published from 1974-1990. This author found no differences in risk of $\mathrm{BrCa}$ among ever- and never-users of oral contraceptives (RR, 1.0; 95\% CI, 1.0-1.1). Rushton and Jones [19], analyzing data from 21 case-control studies from the years 1980-1989, showed that the overall relative risk was 1.12 (95\% CI, 1.05-1.20). Kahlenborn et al. [22] performed a meta-analysis of 34 case control studies of the relationships between OC administration and premenopausal breast cancer published in or after 1980 and found oral contraceptive use was associated with an increased BrCa risk (OR, 1.19; 95\% CI, 1.09-1.29). A pooled analysis made by Collaborative Group on Hormonal Factors in Breast Cancer [21] of data from 54 epidemiological studies, reported in 1996, showed a small increase in the risk of $\mathrm{BrCa}$ with ever use ( $\mathrm{RR}=1.07$ for all studies; $\mathrm{RR}=1.07$ for cohort studies; $\mathrm{RR}=1.02$ for case-control studies with population controls; $R R=1.17$ for case-control studies with hospital controls). Our large standardized meta-analysis conducted on the largest data set from 79 case-control studies published from 1960-2010, showed that ever use of OC is not associated with an increased risk of breast cancer, compared with never using $(\mathrm{OR}=1.1)$.

Evaluation of carcinogenic risks associated with changes in the formulations of OC over the past decades, especially of lowering estrogen content, also remains unclear. In our meta-analysis, based on accepted criteria, we found no increased risk of breast cancer associated with use of low-potency/low estrogen-dose oral contraceptives, a finding which was comparable with earlier high-potency/high-dose preparations. Our results confirmed earlier reports of Collaborative Group on Hormonal Factors in Breast Cancer [112] that among women for whom information was available concerning the OC formulations used, there was no significant variation in the breast cancer risk associated with use specific types and doses of estrogen or progestogen. In a meta-analysis based on different periods of inclusion in the study, Romieu et al. [110] also showed no significant effect of different OC formulations on risk of breast cancer: Cases accrued between 1975-1989-RR $=1.08$, and cases accrued between 1980-1989-RR = 1.06. Rushton and Jones [19], based on the criterion of study ending, adopted a limit of 1982 or earlier versus 1983 or later, demonstrating that low-dose OCs are associated with a greater risk of breast cancer than high-dose regimens, $R R=0.98$ and $R R=1.16$, respectively. These differences were more pronounced, depending on the age of women using OC: $R R=0.90$ and $R R=1.25$, respectively for age $<45$ years, and $R R=1.06$ and $R R=1.00$, respectively for age $\geq 45$ years. Similarly, Kahlenborn et al. [22] also showed an increased risk of premenopausal breast cancer under the influence of low-dose OC $(\mathrm{OR}=1.19)$.

In our meta-analysis, we performed only separate analysis for assessment of relationship between risk of breast cancer and use of older oral contraceptives formulations containing higher doses of estrogen and oral contraceptives using low-estrogen formulations, based on the periods of recruitment to the study, creating two subgroups-the periods of recruitment 1966-1985 and 1986-2010. However, we have not identified different oral contraceptives, because only 14 of the 79 studies included in the meta-analysis reported the type of oral contraceptive used. The analysis of more than half of these studies showed that no statistically significant differences were found in the incidence of breast cancer between cases and controls, depending on the use of different oral contraceptives $[32,43,44,47,50,62,65,94]$. In contrast, White et al., demonstrated that the use of 
high-progestin pills is associated with an increased risk of breast cancer on use for $1-5$ years $(\mathrm{OR}=1.64 ; 95 \% \mathrm{CI}=1.14-2.35)$ or 6 years and above $(\mathrm{OR}=1.61 ; 95 \% \mathrm{CI}=1.0-2.60)[61]$. A study by Rookus et al., found an increased risk for the use of low-dose estrogen pills over 8 years $(\mathrm{RR}=3.0 ; 95 \% \mathrm{CI}=1.5-6.1)$ and norethisterone (acetate) pills over 4 years of use $(\mathrm{RR}=2.0 ; 95 \% \mathrm{CI}=1.0-4.1)$ or ever used desogestrel-containing pills $(\mathrm{RR}=1.7 ; 95 \%$ $\mathrm{CI}=1.1-2.5$ ) [72]. Furthermore, the UK National Case-Control Study has shown an increase in the risk of breast cancer with high estrogen pills over time $(p<0.001)$ [55]. On the other hand, McPherson et al., showed an increased risk of breast cancer when using ethinylestradiol over 48 months before the first pregnancy $(R R=2.62 ; 95 \% C I=1.15-5.95$ [52]. In turn, in the work of Althuis et al., the use of several types of oral contraception was associated with an increased risk of breast cancer [80]. The highest risk was associated with the use of ethynodiol diacetate $(R R=12.0 ; 95 \% C I=2.4-59.2)$, high doses of progestin $(R R=8.11$; $95 \% \mathrm{CI}=2.1-31.6)$, and a combination of high doses of progestin and low doses of estrogen $(\mathrm{RR}=8.07 ; 95 \% \mathrm{CI}=2.1-31.4)$. Finally, the Shapiro et al., study indicated an increased risk of current use of injectable progestogen contraceptives ( $R R=1.6 ; 95 \% C I=1.1-2.3)$ [92].

Results of our meta-analysis did not always disagree with those from previous reports suggesting that certain groups of women have an increased risk of breast cancer related to OC use. We failed to show appreciable differences in the risk of breast cancer between women who had taken OC and those who had not when we sub-grouped women according to menopausal status. Analysis of cancer risk in premenopausal women showed a slight, statistically non-significant increase (OR, 1.06; 95\% CI, 0.92-1.22) and a marginal decrease in postmenopausal women (OR, 0.99; 95\% CI, 0.89-1.10). In contrast, Romieu et al. [110] showed that each use of OC increases, but non-significantly, breast cancer risk among women $\leq 45$ years of age (RR, 1.17; 95\% CI, 0.95-1.45). A similar result was obtained by Delgado et al. [20] $-\mathrm{RR}=1.10$. Rushton and Jones's meta-analysis [19] demonstrated a statistically significant increase in risk in women $<45$ years (RR, 1.16; 95\% CI, 1.07-1.25) and a marginal increase in women $\geq 45$ years ( $R R, 1.03 ; 95 \% \mathrm{CI}, 0.4-1.13$ ). These results were confirmed by Kahlenborn et al. [22] (OR, 1.19; 95\% CI, 1.09-1.29), as well as by Thomas [111] (RR, 1.16; 95\% CI, 1.05-1.28).

The controversy also concerns breast cancer risk in young women taking the pill. In our investigation, among women who started using OC at an early age (before 25 years), we saw a slight decrease in risk of breast cancer (OR 0.91, 95\% CI, 0.83-1.00). By contrast, the meta-analyses that analyzed risk factor for age-related use reported that an early start in taking birth control pills was associated with the risk of cancer. In the Oxford study [21], the authors showed that in users $<20$ years, the RR was 1.22 and for 20-24 years it was 1.04. Delgado-Rodriquez et al. [20] also demonstrated increased risk under 25 years (RR, 1.25; 95\% CI, 1.10-1.44).

The results of our analysis (OR, 1.02) do not confirm previous papers that reported an increased RR in the range of 1.21-1.30 for oral contraception use by nulliparous women $[19,21,22]$. One of the important findings of our meta-analysis, however, was to demonstrate an increased risk of breast cancer among ever users of oral contraceptives before first full-term pregnancy compared with never users (OR, 1.14; 95\% CI. 1.01-1.28). The Oxford pooled analysis found that current OC users had a higher cancer risk (RR, 1.33) [21]. This also confirmed the results of earlier meta-analyses, which showed an increased risk of breast cancer in these women: Romieu et al. [110] $-\mathrm{RR}=1.72$ (95\% CI, 1.36-2.19), DelgadoRodriguez et al. [20]—RR $=1.17$ (95\% CI, 1.06-1.30), Thomas [111] $-\mathrm{RR}=1.4$ (95\% CI, 1.2-1.7), and Kahlenborn et al. [22]—OR = 1.44 (95\% CI, 1.28-1.62).

We revealed in our meta-analysis that $\mathrm{OC}$ use longer than 5 years leads to a modest, but significant increased risk of breast cancer (OR, 1.09; 95\% CI, 1.01-1.18). Romieu et al. [110] also showed that OC use for 10 years or longer increased cancer risk (RR, 1.14; 95\% CI, 0.90-1.42). Similarly, Thomas [111] demonstrated that summary relative risk of breast cancer in women under 45 years who used OC for a long period ( $\geq 10$ years) was 1.42 (95\% $\mathrm{CI}, 1.25-1.63)$. In turn, Rushton and Jones [19] in their meta-analysis estimated the $\mathrm{RR}=1.27$ ( $95 \%$ CI, 1.12-1.44) for use durations of more than 8 years. The Oxford analysis [21] found 
that relative risk of breast cancer by 5-9 years duration of OC use 5-9 years was 1.09, by duration of 10-14 years was 1.6 , and by $\geq 15$ was 1.08 .

Very important discoveries regarding the etiology of breast cancer concern the mutations in the BRCA1/2 genes. However, none of the works in this meta-analysis have tested cases and controls in this regard. Indeed, only Ursin et al., and Marchbanks et al., mentioned in their discussion sections the possible influence of BRCA1 and BRCA2 mutations on the occurrence of breast cancer with the simultaneous use of OC [65,94]. It should also be noted that Beji and Reis analyzed the occurrence of breast cancer in the context of family history, writing that BRCA1/2 mutations are found in most families with a positive history of breast, ovarian, and endometrial cancer [105]. However, they add that the people taking part in the study were not tested for the presence of these mutations. In turn, Mahouri et al., mentioned the need to study the impact of mutations in the BRCA1 and BRCA2 genes on the incidence of breast cancer in Iranian women [103].

In the period analyzed by us (up to 2010), however, there were several papers dealing with the topic of mutations in the BRCA1 and BRCA2 genes. Brohet et al., showed that BRCA1/2 mutation carriers who had ever used oral contraception had a significantly increased risk of breast cancer $(\mathrm{HR}=1.47 ; 95 \% \mathrm{CI}=1.16-1.87)$. Longer use of OC, especially before the first full-term pregnancy, was also associated with an increased risk of breast cancer for both BRCA1 and BRCA2 mutation carriers (HR $=1.49 ; 95 \% \mathrm{CI}=1.05-2.11$ for BRCA1 subjects and $\mathrm{HR}=2.58 ; 95 \% \mathrm{CI}=1.21-5.49$ for $\mathrm{BRCA} 2$ subjects) [113]. Still, in a study by Lee et al., no association was found between the use of oral contraceptives and the risk of breast cancer in carriers of BRCA1 and BRCA2 mutations [114]. Moreover, a study by Milne et al. showed that the use of oral contraceptives for at least 12 months significantly reduced the risk of breast cancer for BRCA1 mutation carriers $(\mathrm{OR}=0.22 ; 95 \%$ $\mathrm{CI}=0.10-0.49)$, but not for BRCA2 mutation carriers (OR $=0.93 ; 95 \% \mathrm{CI}=0.69-1.24)$ [115].

\section{Study Limitations}

In our meta-analysis, significant heterogeneity between studies is observed, although the specific cause of its occurrence across individual studies could not be easily explained.

We were aware of a number of limitations that may affect the interpretation of our results. First, as with all meta-analyses, the validity of the results is limited by the conduct and reporting of the studies from which the data were extracted and pooled. In order to reduce the possibility of publication bias, we limited the search to studies published in indexed journals, and we did not search for non-published studies. Nevertheless, lack of these reports as well as the possibility of not reaching out to all publications in this topic may affect the value of the results [116]. Secondly, the studies contributing to summary estimates are vulnerable to various types of bias. Retrospective self-reporting of users of oral contraceptives was present in the studies we found; this may be associated with overestimation or underestimation of data. Yet another element of bias that must be considered is the possibility to make a mistake in recruitment to the control groups, especially based on hospital populations. Selection bias can also occur when cases are either more or less likely than controls to be selected for study depending on their use of OC. Another possible source of bias present in meta-analysis is the lack of a uniform definition of 'ever' use of oral contraceptives. This term contains a different period of exposure to OC, defined by the various times that pills were started and stopped. This may lead to misclassifications, which may weaken the true association between OC use and breast cancer (as it may occur unequally among cases and controls).

The limitations should also take into account other coexisting factors, such as the use of different oral contraceptives, as well as the presence of unexplored genetic factors, such as BRCA1 and BRCA2 mutations. We are, hence, currently preparing a meta-analysis in which we will examine the effect of BRCA1 and BRCA2 mutations on the occurrence of breast cancer.

A further limitation lies in the fact that most of the studies in this meta-analysis only look at the effect of contraception on breast cancer in general. Moreover, only some studies 
assessed the risk of breast cancer after using different oral contraceptives. These articles were too small a research area for the analyses we undertook. Furthermore, in all of the analyzed studies, participants were not tested for the presence of mutations in the BRCA1 and BRCA2 genes.

Finally, as with other meta-analyses, given that many studies are not included in the evaluation of additional variables or analyzed differing confounders, we were not able to adjust our overall analysis for these risk factors. Thus, we only used crude (not adjusted) study estimates in our meta-analysis. If there were strong effects from confounding factors, the estimates included in the meta-analysis might be biased. A prospective study needs to be conducted to confirm our findings.

\section{Conclusions}

In conclusion, the findings of our meta-analysis and systematic review suggest that oral contraceptives do not appear to increase the risk of breast cancer among women who are taking these preparations. However, OC use before a first full-term pregnancy or using them longer than 5 years can modify the development of the breast cancer.

Supplementary Materials: The following are available online at https://www.mdpi.com/article/10 .3390 /ijerph18094638/s1, Table S1: Quality assessment of included studies based on the NewcastleOttawa Scale score.

Author Contributions: Conceptualization, W.K. and A.B. (Agata Błaszczuk); methodology, W.K., A.B. (Agnieszka Barańska) and M.M.; software, M.J. (Mariola Janiszewska); formal analysis, W.K., M.M. and A.B. (Agata Błaszczuk); investigation, W.K., M.J. (Mariola Janiszewska) and A.B. (Agnieszka Barańska); resources, M.J. (Marian Jędrych); writing-original draft preparation, W.K.; writingreview and editing, W.K. and A.B. (Agata Błaszczuk); visualization, A.B. (Agata Błaszczuk); supervision, W.K., M.P.-D. and M.J. (Marian Jędrych); project administration, A.B. (Agnieszka Barańska) and M.P.-D. All authors have read and agreed to the published version of the manuscript.

Funding: This research received no external funding.

Institutional Review Board Statement: Not applicable.

Informed Consent Statement: Not applicable.

Data Availability Statement: The datasets used or analyzed during the current study are available from the corresponding author on reasonable request.

Conflicts of Interest: The authors declare no conflict of interest.

\section{References}

1. Jemal, A.; Bray, F.; Center, M.M.; Ferlay, J.; Ward, E.; Forman, D. Global cancer statistics. CA Cancer J. Clin. 2011, 61, 69-90. [CrossRef]

2. McPherson, K.; Steel, C.M.; Dixon, J.M. Breast cancer-epidemiology, risk factors, and genetics. BMJ 2000, 321, 624-628. [CrossRef]

3. Althuis, M.D.; Dozier, J.M.; Anderson, W.F.; Devesa, S.S.; Brinton, L.A. Global trends in breast cancer incidence and mortality 1973-1997. Int. J. Epidemiol. 2005, 34, 405-412. [CrossRef] [PubMed]

4. Héry, C.; Ferlay, J.; Boniol, M.; Autier, P. Changes in breast cancer incidence and mortality in middle-aged and elderly women in 28 countries with Caucasian majority populations. Ann. Oncol. 2008, 19, 1009-1018. [CrossRef] [PubMed]

5. Glass, A.G.; Lacey, J.V., Jr.; Carreon, J.D.; Hoover, R.N. Breast cancer incidence, 1980-2006: Combined roles of menopausal hormone therapy, screening mammography, and estrogen receptor status. J. Natl. Cancer Inst. 2007, 99, 1152-1161. [CrossRef] [PubMed]

6. Ravdin, P.M.; Cronin, K.A.; Howlader, N.; Berg, C.D.; Chlebowski, R.T.; Feuer, E.J.; Edwards, B.K.; Berry, D.A. The decrease in breast-cancer incidence in 2003 in the United States. N. Engl. J. Med. 2007, 356, 1670-1674. [CrossRef] [PubMed]

7. Verkooijen, H.M.; Bouchardy, C.; Vinh-Hung, V.; Rapiti, E.; Hartman, M. The incidence of breast cancer and changes in the use of hormone replacement therapy: A review of the evidence. Maturitas 2009, 64, 80-85. [CrossRef] [PubMed]

8. Rossouw, J.E.; Anderson, G.L.; Prentice, R.L.; LaCroix, A.Z.; Kooperberg, C.; Stefanick, M.L.; Jackson, R.D.; Beresford, S.A.A.; Howard, B.V.; Johnson, K.C.; et al. Writing Group for the Women's Health Initiative Investigators. Risks and benefits of estrogen plus progestin in healthy postmenopausal women: Principal results From the Women's Health Initiative randomized controlled trial. JAMA 2002, 288, 321-333.

9. Casey, P.M.; Cerhan, J.R.; Pruthi, S. Oral contraceptive use and risk of breast cancer. Mayo Clin. Proc. 2008, 83, 86-90. [CrossRef] 
10. Breen, N.; Gentleman, J.F.; Schiller, J.S. Update on mammography trends: Comparisons of rates in 2000, 2005, and 2008. Cancer 2011, 117, 2209-2218. [CrossRef]

11. Turnbull, C.; Hodgson, S. Genetic predisposition to cancer. Clin. Med. 2005, 5, 491-498. [CrossRef] [PubMed]

12. Kaaks, R.; Berrino, F.; Key, T.; Rinaldi, S.; Dossus, L.; Biessy, C.; Secreto, G.; Amiano, P.; Bingham, S.; Boeing, H.; et al. Serum sex steroids in premenopausal women and breast cancer risk within the European Prospective Investigation into Cancer and Nutrition (EPIC). J. Natl. Cancer Inst. 2005, 97, 755-765. [CrossRef] [PubMed]

13. Kaaks, R.; Rinaldi, S.; Key, T.J.; Berrino, F.; Peeters, P.M.H.; Biessy, C.; Dossus, L.; Lukanova, A.; Bingham, S.; Khaw, K.-T.; et al Postmenopausal serum androgens, oestrogens and breast cancer risk: The European prospective investigation into cancer and nutrition. Endocr. Relat. Cancer 2005, 12, 1071-1082. [CrossRef]

14. Mansour, D.; Inki, P.; Gemzell-Danielsson, K. Efficacy of contraceptive methods: A review of the literature. Eur. J. Contracept. Reprod. Health Care 2010, 15, 4-16. [CrossRef]

15. IARC Combined estrogen-progestogen contraceptives and combined estrogen-progestogen menopausal therapy. IARC Monogr. Eval. Carcinog. Risks Hum. 2007, 91, 1-528.

16. Cibula, D.; Gompel, A.; Mueck, A.O.; La Vecchia, C.; Hannaford, P.C.; Skouby, S.O.; Zikan, M.; Dusek, L. Hormonal contraception and risk of cancer. Hum. Reprod. Update 2010, 16, 631-650. [CrossRef]

17. Kahlenborn, C.; Modugno, F.; Severs, W.B. Oral contraceptives and breast cancer. Mayo Clin. Proc. 2008, 83, 849-851. [CrossRef]

18. Hawley, W.; Nuovo, J.; DeNeef, C.P.; Carter, P. Do oral contraceptive agents affect the risk of breast cancer? A meta-analysis of the case-control reports. J. Am. Board Fam. Pract. 1993, 6, 123-135.

19. Rushton, L.; Jones, D.R. Oral contraceptive use and breast cancer risk: A meta-analysis of variations with age at diagnosis, parity and total duration of oral contraceptive use. Br. J. Obs. Gynaecol. 1992, 99, 239-246. [CrossRef]

20. Delgado-Rodriguez, M.; Sillero-Arenas, M.; Rodriguez-Contreras, R.; López Gigosos, R.; Gàlvez Vargas, R. Oral contraceptives and breast cancer. A meta-analysis. Rev. Epidemiol. Sante Publique 1991, 39, 165-181.

21. Collaborative Group on Hormonal Factors in Breast Cancer. Breast cancer and hormonal contraceptives: Collaborative reanalysis of individual data on 53297 women with breast cancer and 100239 women without breast cancer from 54 epidemiological studies. Lancet 1996, 347, 1713-1727. [CrossRef]

22. Kahlenborn, C.; Modugno, F.; Potter, D.M.; Severs, W.B. Oral contraceptive use as a risk factor for premenopausal breast cancer: A meta-analysis. Mayo Clin. Proc. 2006, 81, 1290-1302. [CrossRef] [PubMed]

23. Gerstman, B.B.; Gross, T.P.; Kennedy, D.L.; Bennett, R.C.; Tomita, D.K.; Stadel, B.V. Trends in the content and use of oral contraceptives in the United States, 1964-1988. Am. J. Public Health 1991, 81, 90-96. [CrossRef]

24. Westhoff, C. Trends in oral contraceptive development and utilization: Looking to the future. Contracept. Rep. 1997, 7, 4-13.

25. Heinemann, K.; Moehner, S.; Lewis, M.; Assmann, A.; Garbe, E.; Heinemann, L.A. Trend der OC-Nutzung in einer Deutschen Frauen-Kohorte 1980-1999. Ergebnisse der Deutschen Kohortenstudie zur Frauengesundheit. Zent. Gynakol 2002, 124, 128-131. [CrossRef]

26. Thorogood, M.; Vessey, M.P. Trends in use of oral contraceptives in Britain. Brit. J. Fam. Plan. 1990, 16, 11-53.

27. Stang, A. Critical evaluation of the Newcastle-Ottawa scale for the assessment of the quality of nonrandomized studies in metaanalyses. Eur. J. Epidemiol. 2010, 25, 603-605. [CrossRef]

28. DerSimonian, R.; Laird, N. Meta-analysis in clinical trials. Control. Clin. Trials 1986, 7, 177-188. [CrossRef]

29. Higgins, J.P.; Thompson, S.G. Quantifying heterogeneity in a meta-analysis. Stat. Med. 2002, 21, 1539-1558. [CrossRef]

30. Higgins, J.P.; Thompson, S.G.; Deeks, J.J.; Altman, D.G. Measuring inconsistency in meta-analyses. BMJ 2003, 327, 557-560. [CrossRef]

31. Hennekens, C.H.; Speizer, F.E.; Lipnick, R.J.; Rosner, B.; Bain, C.; Belanger, C.; Stampfer, M.J.; Willett, W.; Peto, R. A case-control study of oral contraceptive use and breast cancer. J. Natl. Cancer Inst. 1984, 72, 39-42. [CrossRef] [PubMed]

32. Vessey, M.; Baron, J.; Doll, R.; McPherson, K.; Yeates, D. Oral contraceptives and breast cancer: Final report of an epidemiological study. Br. J. Cancer 1983, 47, 455-462. [CrossRef] [PubMed]

33. Wynder, E.L.; MacCornack, F.A.; Stellman, S.D. The epidemiology of breast cancer in 785 United States Caucasian women. Cancer 1978, 41, 2341-2354. [CrossRef]

34. Sartwell, P.E.; Arthes, F.G.; Tonascia, J.A. Exogenous hormones, reproductive history, and breast cancer. J. Natl. Cancer Inst. 1977, 59, 1589-1592. [CrossRef]

35. Paffenbarger, R.S., Jr.; Kampert, J.B.; Chang, H.G. Characteristics that predict risk of breast cancer before and after the menopause. Am. J. Epidemiol. 1980, 112, 258-268. [CrossRef]

36. Lees, A.W.; Burns, P.E.; Grace, M. Oral contraceptives and breast disease in premenopausal northern Albertan women. Int. J. Cancer 1978, 22, 700-707. [CrossRef]

37. Ravnihar, B.; Seigel, D.G.; Lindtner, J. An epidemiologic study of breast cancer and benign breast neoplasia in relation to the oral contraceptive and estrogen use. Eur. J. Cancer 1979, 15, 395-405. [CrossRef]

38. Pike, M.C.; Henderson, B.E.; Casagrande, J.T.; Rosario, I.; Gray, G.E. Oral contraceptive use and early abortion as risk factors for breast cancer in young women. Br. J. Cancer 1981, 43, 72-76. [CrossRef]

39. Stanford, J.L.; Brinton, L.A.; Hoover, R.N. Oral contraceptives and breast cancer: Results from an expanded case-control study. Br. J. Cancer 1989, 60, 375-381. [CrossRef] 
40. Janerich, D.T.; Polednak, A.P.; Glebatis, D.M.; Lawrence, C.E. Breast cancer and oral contraceptive use: A case-control study. J. Chronic Dis. 1983, 36, 639-646. [CrossRef]

41. Lubin, J.H.; Burns, P.E.; Blot, W.J.; Lees, A.W.; May, C.; Morris, L.E.; Fraumeni, J.F., Jr. Risk factors for breast cancer in women in northern Alberta, Canada, as related to age at diagnosis. J. Natl. Cancer Inst. 1982, 68, 211-217.

42. Schildkraut, J.M.; Hulka, B.S.; Wilkinson, W.E. Oral contraceptives and breast cancer: A case-control study with hospital and community controls. Obs. Gynecol. 1990, 76, 395-402. [CrossRef]

43. Harris, N.V.; Weiss, N.S.; Francis, A.M.; Polissar, L. Breast cancer in relation to patterns of oral contraceptive use. Am. J. Epidemiol. 1982, 116, 643-651. [CrossRef]

44. Rosenberg, L.; Palmer, J.R.; Rao, R.S.; Zauber, A.G.; Strom, B.L.; Warshauer, M.E.; Harlap, S.; Shapiro, S. Case-control study of oral contraceptive use and risk of breast cancer. Am. J. Epidemiol. 1996, 143, 25-37. [CrossRef]

45. Palmer, J.R.; Rosenberg, L.; Rao, R.S.; Strom, B.L.; Warshauer, M.E.; Harlap, S.; Zauber, A.; Shapiro, S. Oral contraceptive use and breast cancer risk among African-American women. Cancer Causes Control 1995, 6, 321-331. [CrossRef]

46. Gomes, A.L.; Guimarães, M.D.; Gomes, C.C.; Chaves, I.G.; Gobbi, H.; Camargos, A.F. A case-control study of risk factors for breast cancer in Brazil, 1978-1987. Int. J. Epidemiol. 1995, 24, 292-299. [CrossRef]

47. Jick, S.S.; Walker, A.M.; Stergachis, A.; Jick, H. Oral contraceptives and breast cancer. Br. J. Cancer 1989, 59, 618-621. [CrossRef]

48. Harris, R.E.; Zang, E.A.; Wynder, E.L. Oral contraceptives and breast cancer risk: A case-control study. Int. J. Epidemiol. 1990, 19, 240-246. [CrossRef]

49. Olsson, H.; Möller, T.R.; Ranstam, J. Early oral contraceptive use and breast cancer among premenopausal women: Final report from a study in southern Sweden. J. Natl. Cancer Inst. 1989, 81, 1000-1004. [CrossRef]

50. Ravnihar, B.; Primic-Žakelj, M.; Košmelj, K.; Stare, J. A case-control study of breast cancer in relation to oral contraceptive use in Slovenia. Neoplasia 1988, 35, 109-121.

51. Talamini, R.; La Vecchia, C.; Franceschi, S.; Colombo, F.; Decarli, A.; Grattoni, E.; Grigoletto, E.; Tognoni, G. Reproductive and hormonal factors and breast cancer in a Northern Italian population. Int. J. Epidemiol. 1985, 14, 70-74. [CrossRef] [PubMed]

52. McPherson, K.; Vessey, M.P.; Neil, A.; Doll, R.; Jones, L.; Roberts, M. Early oral contraceptive use and breast cancer: Results of another case-control study. Br. J. Cancer 1987, 56, 653-660. [CrossRef] [PubMed]

53. Wingo, P.A.; Lee, N.C.; Ory, H.W.; Beral, V.; Peterson, H.B.; Rhodes, P. Age specific differences in the relationship between oral contraceptive use and breast cancer. Obs. Gynecol. 1991, 78, 161-170. [CrossRef]

54. Ellery, C.; MacLennan, R.; Berry, G.; Shearman, R.P. A case-control study of breast cancer in relation to the use of steroid contraceptive agents. Med. J. Aust. 1986, 144, 173-176. [CrossRef]

55. UK National Case-control Study Group. Oral contraceptive use and breast cancer risk in young women. Lancet 1989, 1, 973-982.

56. Lee, N.C.; Rosero-Bixby, L.; Oberle, M.W.; Grimaldo, C.; Whatley, A.S.; Rovira, E.Z. A case-control study of breast cancer and hormonal contraception in Costa Rica. J. Natl. Cancer Inst. 1987, 79, 1247-1254.

57. Rohan, T.E.; McMichael, A.J. Oral contraceptive agents and breast cancer: A population-based case-control study. Med. J. Aust. 1988, 149, 520-526. [CrossRef]

58. WHO Collaborative Study of Neoplasia and Steroid Contraceptives. Breast cancer and combined oral contraceptives: Results from a multinational study. Br. J. Cancer 1990, 61, 110-119. [CrossRef]

59. Rosenberg, L.; Palmer, J.R.; Clarke, E.A.; Shapiro, S. A case-control study of the risk of breast cancer in relation to oral contraceptive use. Am. J. Epidemiol. 1992, 136, 1437-1444. [CrossRef]

60. Tavani, A.; Negri, E.; Franceschi, S.; Parazzini, F.; La Vecchia, C. Oral contraceptives and breast cancer in North Italy. Final report from a case-control study. Br. J. Cancer 1993, 68, 568-571. [CrossRef]

61. White, E.; Malone, K.E.; Weiss, N.S.; Daling, J.R. Breast cancer among young U.S. women in relation to oral contraceptive use. J. Natl. Cancer Inst. 1994, 86, 505-514. [CrossRef]

62. Ewertz, M. Oral contraceptives and breast cancer risk in Denmark. Eur. J. Cancer 1992, 28A, 1176-1781. [CrossRef]

63. Ursin, G.; Wu, A.H.; Hoover, R.N.; West, D.W.; Nomura, A.M.; Kolonel, L.N.; Pike, M.C.; Ziegler, R.G. Breast cancer and oral contraceptive use in Asian-American women. Am. J. Epidemiol. 1999, 150, 561-567. [CrossRef]

64. Paul, C.; Skegg, D.C.G. Spears GF. Oral contraceptive use and risk of breast cancer in older women (New Zealand). Cancer Causes Control 1995, 6, 485-591. [CrossRef]

65. Ursin, G.; Ross, R.K.; Sullivan-Halley, J.; Hanisch, R.; Henderson, B.; Bernstein, L. Use of oral contraceptives and risk of breast cancer in young women. Breast Cancer Res. Treat. 1998, 50, 175-184. [CrossRef]

66. Clavel, F.; Andrieu, N.; Gairard, B.; Brémond, A.; Piana, L.; Lansac, J.; Bréart, G.; Rumeau-Rouquette, C.; Flamant, R.; Renaud, R. Oral contraceptives and breast cancer: A French case-control study. Int. J. Epidemiol. 1991, 20, 32-38. [CrossRef]

67. Weinstein, A.L.; Mahoney, M.C.; Nasca, P.C.; Leske, M.C.; Varma, A.O. Breast cancer risk and oral contraceptive use: Results from a large case-control study. Epidemiology 1991, 2, 353-358. [CrossRef]

68. Lund, E.; Meirik, O.; Adami, H.O.; Bergstrøm, R.; Christoffersen, T.; Bergsjø, P. Oral contraceptive use and premenopausal breast cancer in Sweden and Norway: Possible effects of different pattern of use. Int. J. Epidemiol. 1989, 18, 527-532. [CrossRef]

69. Yuan, J.M.; Yu, M.C.; Ross, R.K.; Gao, Y.T.; Henderson, B.E. Risk factors for breast cancer in Chinese women in Shanghai. Cancer Res. 1988, 48, 1949-1953.

70. Wang, Q.S.; Ross, R.K.; Yu, M.C.; Ning, J.P.; Henderson, B.E.; Kimm, H.T. A case-control study of breast cancer in Tianjin, China. Cancer Epidemiol. Biomark. Prev. 1992, 1, 435-439. 
71. Viladiu, P.; Izquierdo, A.; de Sanjosé, S.; Bosch, F.X. A breast cancer case-control study in Girona, Spain. Endocrine, familial and lifestyle factors. Eur. J. Cancer Prev. 1996, 5, 329-335. [CrossRef]

72. Rookus, M.A.; van Leeuwen, F.E. Netherlands Oral Contraceptives and Breast Cancer Study Group. Oral contraceptives and risk of breast cancer in women aged 20-54 years. Lancet 1994, 344, 844-851. [CrossRef]

73. Lee, H.P.; Gourley, L.; Duffy, S.W.; Estève, J.; Lee, J.; Day, N.E. Risk factors for breast cancer by age and menopausal status: A case-control study in Singapore. Cancer Causes Control 1992, 3, 313-322. [CrossRef]

74. Primic-Žakelj, M.; Evstifeeva, T.; Ravnihar, B.; Boyle, P. Breast-cancer risk and oral contraceptive use in Slovenian women aged 25 to 54. Int. J. Cancer 1995, 62, 414-420. [CrossRef]

75. Rossing, M.A.; Stanford, J.L.; Weiss, N.S.; Habel, L.A. Oral contraceptive use and risk of breast cancer in middle-aged women. Am. J. Epidemiol. 1996, 144, 161-164. [CrossRef]

76. Newcomb, P.A.; Longnecker, M.P.; Storer, B.E.; Mittendorf, R.; Baron, J.; Clapp, R.W.; Trentham-Dietz, A.; Willett, W.C. Recent oral contraceptive use and risk of breast cancer (United States). Cancer Causes Control 1996, 7, 525-532. [CrossRef]

77. Lipworth, L.; Katsouyanni, K.; Stuver, S.; Samoli, E.; Hankinson, S.E.; Trichopoulos, D. Oral contraceptives, menopausal estrogens, and the risk of breast cancer: A case-control study in Greece. Int. J. Cancer 1995, 62, 548-551. [CrossRef]

78. Kishk, N.A. Breast cancer in relation to some reproductive factors. J. Egypt Public Health Assoc. 1999, 74, 547-566.

79. Levi, F.; Lucchini, F.; Pasche, C.; La Vecchia, C. Oral contraceptives, menopausal hormone replacement treatment and breast cancer risk. Eur. J. Cancer Prev. 1996, 5, 259-266. [CrossRef]

80. Althuis, M.D.; Brogan, D.R.; Coates, R.J.; Daling, J.R.; Gammon, M.D.; Malone, K.E.; Schoenberg, J.B.; Brinton, L.A. Hormonal content and potency of oral contraceptives and breast cancer risk among young women. Br. J. Cancer 2003, 88, 50-57. [CrossRef]

81. Bustan, M.N.; Coker, A.L.; Addy, C.L.; Macera, C.A.; Greene, F.; Sampoerno, D. Oral contraceptive use and breast cancer in Indonesia. Contraception 1993, 47, 241-249. [CrossRef]

82. La Vecchia, C.; Negri, E.; Franceschi, S.; Talamini, R.; Amadori, D.; Filiberti, R.; Conti, E.; Montella, M.; Veronesi, A.; Parazzini, F.; et al. Oral contraceptives and breast cancer: A cooperative Italian study. Int. J. Cancer 1995, 60, 163-167. [CrossRef] [PubMed]

83. McCredie, M.R.; Dite, G.S.; Giles, G.G.; Hopper, J.L. Breast cancer in Australian women under the age of 40. Cancer Causes Control 1998, 9, 189-198. [CrossRef] [PubMed]

84. Adebamowo, C.A.; Adekunle, O.O. Case-controlled study of the epidemiological risk factors for breast cancer in Nigeria. Br. J. Surg. 1999, 86, 665-668. [CrossRef] [PubMed]

85. Traina, A.; Cusimano, R.; Liquori, M.; Calabria, C.; Biglia, N.; Ponzone, R.; Sgrò, L.; Sismondi, P. Oral contraceptive use and breast cancer risk in areas with different incidence: A case-control study among young women. Ann. N. Y. Acad. Sci. 1996, 784, 564-569. [CrossRef] [PubMed]

86. Yang, P.S.; Yang, T.L.; Liu, C.L.; Wu, C.W.; Shen, C.Y. A case-control study of breast cancer in Taiwan-a low-incidence area. Br. J. Cancer 1997, 75, 752-756. [CrossRef]

87. Chie, W.C.; Li, C.Y.; Huang, C.S.; Chang, K.J.; Yen, M.L.; Lin, R.S. Oral contraceptives and breast cancer risk in Taiwan, a country of low incidence of breast cancer and low use of oral contraceptives. Int. J. Cancer 1998, 77, 219-223. [CrossRef]

88. Hall, I.J.; Moorman, P.G.; Millikan, R.C.; Newman, B. Comparative analysis of breast cancer risk factors among African-American women and White women. Am. J. Epidemiol. 2005, 161, 40-51. [CrossRef]

89. Croghan, I.T.; Pruthi, S.; Hays, J.T.; Cha, S.; Johnson, R.E.; Kosel, M.; Morris, R.; Hurt, R.D. The role of smoking in breast cancer development: An analysis of a Mayo Clinic cohort. Breast J. 2009, 15, 489-495. [CrossRef]

90. Magnusson, C.M.; Persson, I.R.; Baron, J.A.; Ekbom, A.; Bergström, R.; Adami, H.O. The role of reproductive factors and use of oral contraceptives in the aetiology of breast cancer in women aged 50 to 74 years. Int. J. Cancer 1999, 80, 231-236. [CrossRef]

91. Rosenberg, L.; Zhang, Y.; Coogan, P.F.; Strom, B.L.; Palmer, J.R. A case-control study of oral contraceptive use and incident breast cancer. Am. J. Epidemiol. 2009, 169, 473-479. [CrossRef]

92. Shapiro, S.; Rosenberg, L.; Hoffman, M.; Truter, H.; Cooper, D.; Rao, S.; Dent, D.; Gudgeon, A.; van Zyl, J.; Katzenellenbogen, J.; et al. Risk of breast cancer in relation to the use of injectable progestogen contraceptives and combined estrogen/progestogen contraceptives. Am. J. Epidemiol. 2000, 151, 396-403. [CrossRef]

93. Price, M.A.; Tennant, C.C.; Smith, R.C.; Kennedy, S.J.; Butow, P.N.; Kossoff, M.B.; Dunn, S.M. Predictors of breast cancer in women recalled following screening. Aust. N. Z. J. Surg. 1999, 69, 639-646. [CrossRef]

94. Marchbanks, P.A.; McDonald, J.A.; Wilson, H.G.; Folger, S.G.; Mandel, M.G.; Daling, J.R.; Bernstein, L.; Malone, K.E.; Ursin, G.; Strom, B.L.; et al. Oral contraceptives and the risk of breast cancer. N. Engl. J. Med. 2002, 346, 2025-2032. [CrossRef]

95. Tessaro, S.; Béria, J.U.; Tomasi, E.; Barros, A.J.D. Contraceptivos orais e cancer de mama: Estudo de casos e controles. Rev. Saúde Pública 2001, 35, 32-38. [CrossRef]

96. Shantakumar, S.; Terry, M.B.; Paykin, A.; Teitelbaum, S.L.; Britton, J.A.; Moorman, P.G.; Kritchevsky, S.B.; Neugut, A.I.; Gammon, M.D. Age and menopausal effects of hormonal birth control and hormone replacement therapy in relation to breast cancer risk. Am. J. Epidemiol. 2007, 165, 1187-1198. [CrossRef]

97. Ebrahimi, M.; Vahdaninia, M.; Montazeri, A. Risk factors for breast cancer in Iran: A case-control study. Breast Cancer Res. 2002, 4, R10. [CrossRef]

98. Kuru, B.; Ozaslan, C.; Ozdemir, P.; Dinç, S.; Camlibel, M.; Alagöl, H. Risk factors for breast cancer in Turkish women with early pregnancies and long-lasting lactation-a case-control study. Acta Oncol. 2002, 41, 556-561. [CrossRef] 
99. Hadjisavvas, A.; Loizidou, M.A.; Middleton, N.; Michael, T.; Papachristoforou, R.; Kakouri, E.; Daniel, M.; Papadopoulos, P.; Malas, S.; Marcou, Y.; et al. An investigation of breast cancer risk factors in Cyprus: A case control study. BMC Cancer 2010, $10,447$. [CrossRef] [PubMed]

100. Lawlor, D.A.; Ebrahim, S.; Smith, G.D. Smoking before the birth of a first child is not associated with increased risk of breast cancer: Findings from the British Women's Heart and Health Cohort Study and a meta-analysis. Br. J. Cancer 2004, 91, 512-518. [CrossRef]

101. Sweeney, C.; Giuliano, A.R.; Baumgartner, K.B.; Byers, T.; Herrick, J.S.; Edwards, S.L.; Slattery, M.L. Oral injected implanted contraceptives breast cancer risk among, U.S. Hispanic and non-Hispanic white women. Int. J. Cancer 2007, 121, $2517-2523$. [CrossRef] [PubMed]

102. Ozmen, V.; Ozcinar, B.; Karanlik, H.; Cabioglu, N.; Tukenmez, M.; Disci, R.; Ozmen, T.; Igci, A.; Muslumanoglu, M.; Kecer, M.; et al. Breast cancer risk factors in Turkish women-a University Hospital based nested case control study. World J. Surg. Oncol. 2009, 7, 37. [CrossRef] [PubMed]

103. Mahouri, K.; Dehghani Zahedani, M.; Zare, S. Breast cancer risk factors in south of Islamic Republic of Iran: A case-control study. East. Mediterr. Health J. 2007, 13, 1265-1273. [CrossRef] [PubMed]

104. Norsa'adah, B.; Rusli, B.N.; Imran, A.K.; Naing, I.; Winn, T. Risk factors of breast cancer in women in Kelantan, Malaysia. Singap. Med. J. 2005, 46, 698-705.

105. Beji, N.K.; Reis, N. Risk factors for breast cancer in Turkish women: A hospital-based case-control study. Eur. J. Cancer Care 2007, 16, 178-184. [CrossRef]

106. Kamarudin, R.; Shah, S.A.; Hidayach, N. Lifestyle factors and breat cancer: A case-control study in Kuala Lumpur, Malaysia. Asian Pac. J. Cancer Prev. 2006, 7, 51-54.

107. Dinger, J.C.; Heinemann, L.A.J.; Möhner, S.; Thai, D.M.; Assman, A. Breast cancer risk associated with different HRT formulations: A register-based case-control study. BMC Women's Health 2006, 6, 13. [CrossRef]

108. Yavari, P.; Mosavizadeh, M.; Sadrol-Hefazi, B.; Mehrabi, Y. Reproductive characteristics and the risk of breast cancer-a case-control study in Iran. Asian Pac. J. Cancer Prev. 2005, 6, 370-375.

109. Faheem, M.; Khurram, M.; Jafri, I.A.; Mehmood, H.; Hasan, Z.; Iqbal, G.S.; Maqsood, F.; Jafri, S.R.A. Risk factors for breast cancer in patients treated at NORI Hospital, Islamabad. J. Pak. Med. Assoc. 2007, 57, 242-245.

110. Romieu, I.; Berlin, J.A.; Colditz, G. Oral contraceptives and breast cancer. Review and meta-analysis. Cancer 1990, 66, $2253-2263$. [CrossRef]

111. Thomas, D.B. Oral contraceptives and breast cancer: Review of the epidemiologic literature. Contraception 1991, 43, 597-642. [CrossRef]

112. Collaborative Group on Hormonal Factors in Breast Cancer. Breast cancer and hormonal contraceptives: Further results. Contraception 1996, 54, 1S-106S. [CrossRef]

113. Brohet, R.M.; Goldgar, D.E.; Easton, D.F.; Antoniou, A.C.; Andrieu, N.; Chang-Claude, J.; Peock, S.; Eeles, R.A.; Cook, M.; Chu, C.; et al. Oral Contraceptives and Breast Cancer Risk in the International BRCA1/2 Carrier Cohort Study: A Report from EMBRACE, GENEPSO, GEO-HEBON, and the IBCCS Collaborating Group. J. Clin. Oncol. 2007, 25, 3831-3836. [CrossRef]

114. Lee, E.; Ma, H.; McKean-Cowdin, R.; Van Den Berg, D.; Bernstein, L.; Henderson, B.E.; Ursin, G. Effect of Reproductive Factors and Oral Contraceptives on Breast Cancer Risk in BRCA1/2 Mutation Carriers and Noncarriers: Results from a Population-Based Study. Cancer Epidemiol. Biomark. Prev. 2008, 17, 3170-3178. [CrossRef]

115. Milne, R.L.; Knight, J.A.; John, E.M.; Dite, G.S.; Balbuena, R.; Ziogas, A.; Andrulis, I.L.; West, D.W.; Li, P.F.; Southey, M.C.; et al. Oral Contraceptive Use and Risk of Early-Onset Breast Cancer in Carriers and Noncarriers of BRCA1 and BRCA2 Mutations. Cancer Epidemiol. Biomark. Prev. 2005, 14, 350-356. [CrossRef]

116. Petitti, D. Meta-Analysis, Decision, Analysis, and Cost-Effectiveness Analysis; Oxford University Press: New York, NY, USA, 2000. 